\title{
Modeling of population kinetics of plasmas that are not in local thermodynamic equilibrium, using a versatile collisional-radiative model based on analytical rates
}

\author{
R. Florido, ${ }^{1,2}$ R. Rodríguez, ${ }^{1,2}$ J. M. Gil,, ${ }^{1,2}$ J. G. Rubiano, ${ }^{1,2}$ P. Martel, ${ }^{1,2}$ E. Mínguez, ${ }^{2}$ and R. C. Mancini ${ }^{3}$ \\ ${ }^{1}$ Departamento de Física, Universidad de Las Palmas de Gran Canaria, Las Palmas de Gran Canaria 35017, Spain \\ ${ }^{2}$ Instituto de Fusión Nuclear, Universidad Politécnica de Madrid, Madrid 28006, Spain \\ ${ }^{3}$ Department of Physics, University of Nevada, Reno, Nevada 89557-0058, USA
}

(Received 5 August 2009; published 4 November 2009)

\begin{abstract}
We discuss the modeling of population kinetics of nonequilibrium steady-state plasmas using a collisionalradiative model and code based on analytical rates (ABAKO). ABAKO can be applied to low-to-high $Z$ ions for a wide range of laboratory plasma conditions: coronal, local thermodynamic equilibrium or nonlocal thermodynamic equilibrium, and optically thin or thick plasmas. ABAKO combines a set of analytical approximations to atomic rates, which yield substantial savings in computer running time, still comparing well with more elaborate codes and experimental data. A simple approximation to calculate the electron capture cross section in terms of the collisional excitation cross section has been adapted to work in a detailed-configurationaccounting approach, thus allowing autoionizing states to be explicitly included in the kinetics in a fast and efficient way. Radiation transport effects in the atomic kinetics due to line trapping in the plasma are taken into account via geometry-dependent escape factors. Since the kinetics problem often involves very large sparse matrices, an iterative method is used to perform the matrix inversion. In order to illustrate the capabilities of the model, we present a number of results which show that the ABAKO compares well with customized models and simulations of ion population distribution. The utility of ABAKO for plasma spectroscopic applications is also outlined.
\end{abstract}

DOI: 10.1103/PhysRevE.80.056402

PACS number(s): 52.25.Dg, 52.20.-j, 52.25.Os, 52.57.-z

\section{INTRODUCTION}

In many research areas such as astrophysics, x-ray lasers, inertial and magnetic confinement fusion, and extreme ultraviolet (EUV) lithography, accurate calculation of the populations of atomic levels is required since they are needed in the determination of the radiative properties for hydrodynamic simulations and spectroscopic diagnostics. In this regard, a review of applications of population kinetics is given in [1].

At high densities, when the plasma approaches local thermodynamic equilibrium (LTE), the populations are calculated by means of the Saha-Boltzmann equations. On the other hand, in the low-density limit, coronal equilibrium (CE) can be assumed and, therefore, these quantities are evaluated using the $\mathrm{CE}$ equations. For arbitrary densities the level populations are determined from the solution of a system of collisional-radiative (CR) rate equations, i.e., the nonLTE (NLTE) regime. This set of kinetic rate equations need to be solved coupling atomic configurations, free electrons, and photons. It is important to consider a sufficiently large number of energy levels in each ionization stage; this leads to the resolution of large linear systems which become sometimes unmanageable and thus approximations must be made. This fact has led to the development of numerous collisionalradiative models and codes since the early proposals described in [2-4].

The first point to consider is the complexity of the atomic model that underlies the CR model. The most detailed level of information that can be used in the determination of the population distributions is usually referred to as the detailedlevel-accounting (DLA) approach, where each atomic level is explicitly included and the solution of a level-by-level kinetic model is required. This approach is considered prac- tical for elements with low to medium atomic number $(Z<30)$ [5]. Codes such as ATOMIC [6], HULLAC [7], NOMAD, [8] and SCRAM [9] work in this approximation. As the atomic number increases, the amount of atomic data involved increases accordingly and the DLA approach becomes impractical and sometimes unnecessary [10]. Thus, for high-Z elements, a configuration-based kinetic model, i.e., a detailed-configuration-accounting (DCA) model is the usual approach. In the literature there are statistical methods to reduce the detail of the atomic description by grouping levels into configurations (resulting in a DCA model) $[11,12]$ or superconfigurations [13]. These methods have shown to be very efficient when they are combined with unresolved transition array (UTA) [14], spin-orbit split array (SOSA) [15] and/or supertransition array [16] formalisms. In these approaches both the amount of atomic data and the number of rate equations are noticeably reduced due to the configuration or superconfiguration average. Codes such as AVERROES/ TRANSPEC [17] or SCROLL [18] use these statistical schemes. However, models based on averages may lack the accuracy to describe isolated levels or transitions correctly. A possible improvement is the definition of effective temperatures inside each statistical group (configuration or superconfiguration), where the detailed level population inside each group is obtained from the group population and the effective temperature, as in the MOST code [19]. Another alternative relies on hybrid models that mix detailed and average descriptions $[5,20]$ or the reduced DCA model [21]. An important goal is to optimize the speed of calculation without loosing significant accuracy. In this connection, RADIOM [22] introduces an extended Saha equation valid in NLTE by means of the definition of an effective ionization temperature, THERMOS [23] and SCAALP [24] employ an average atom, and FLYCHK [25] 
uses a screened hydrogenic model for obtaining the energylevel structure.

A critical issue is the selection of methods and expressions employed for the rate coefficients of the atomic processes included in the CR model. In principle, they should be obtained based on atomic structure and scattering calculations. However, large kinetic calculations require a massive evaluation of rate coefficients. On the other hand, in the literature there are available analytical expressions for the rate coefficients or cross sections of the most relevant atomic processes, although no one of them stands out for its high accuracy. However, due to the complexity of the problem considered, it is necessary to reach a compromise between accuracy and computational viability. Hence, for example, ATOMIC, AVERROES/TRANSPEC, or HULLAC performs quantum-mechanical calculations of the collisional and radiative transition probabilities, FLYCHK employs analytical expressions, and NOMAD and SCRAM often rely on rigorous calculations or accurate online databases for low-lying levels and analytical formulas for the highly excited levels.

There is also the problem of the radiative transfer. For optically thick plasmas the radiative transfer equation is coupled to the rate equations in the $\mathrm{CR}$ model. The radiation transport is important, for instance, in the study of planetary and stellar atmospheres [26] and also in the population balance and spectral properties of laser generated dense plasmas [27]. However, the exact resolution of the radiative transfer equation for each radiative transition in the plasma involves a huge computational cost. Furthermore, due to the coupling with the rate equations, the calculation requires an iterative procedure until the convergence is achieved. For this reason, we can find different ways for dealing with the radiative transport under NLTE conditions [26,28-34]. There are also methods, based on the escape factor formalism, that avoid the explicit solution of the radiative transfer equation and can be applied to homogeneous [35-37] or nonhomogeneous plasmas [38-40].

According to the explained above, there are many sources of discrepancies among different models. The NLTE kinetics workshops [41-44] have focused on comparisons among CR codes for specific cases. These meetings have contributed to identify ranges of plasma conditions where population kinetics is well established, as it happens near closed shell configurations, as well as situations where significant discrepancies occur.

In this paper we discuss the analytical expressions based kinetics code (ABAKO), a CR model to determine the population distribution of atomic levels and radiative properties of steady-state plasmas. Special care was taken to achieve an optimal compromise between accuracy and computational cost. ABAKO combines a set of simple analytical models which yield substantial savings of computer time, still providing good comparisons with more elaborate codes and experimental data. ABAKO has a built-in atomic model that provides atomic structure data in the DCA approach based on a parametric potential, but it can also work with an external source of atomic data. ABAKO employs analytical expressions to compute the rate coefficients of the atomic processes involved in the kinetics and the radiation transport effects are taken into account by means of an escape factor. These fea- tures make ABAKO a versatile CR model which can be applied to low-to-high $Z$ ions for a wide range of laboratory plasma conditions: coronal, LTE or NLTE, and optically thin or thick plasmas. This paper is organized as follows. Section II is devoted to describe the different components of the model, paying particular attention to the CR module. In order to show the capabilities of ABAKO, in Sec. III a wide set of results and comparisons are presented. Finally, conclusions and remarks are discussed in Sec. IV.

\section{MODEL DESCRIPTION}

The objective of ABAKO CR atomic kinetics modeling is to compute atomic level populations in plasma ions for a broad range of plasma environmental conditions. The essential components of a CR model include a description of the atomic levels and the processes that couple those levels. With these two components, a CR model can determine the population of any atom or ion in a given level. The population distribution can be used to obtain the plasma ionization balance and the opacity and emissivity.

\section{A. Atomic structure module}

\section{Determination of the atomic data}

To obtain the atomic data required for kinetic calculations ABAKO has a built-in model that follows a relativistic DCA approach within the central field approximation. As the atomic model by itself has been the aim of several previous works, here we briefly summarize the main points and its application to ABAKO.

Let us consider an ion with atomic number $Z$ and charge $\zeta$. In the DCA context, each bound state $\zeta m$ is associated with a particular relativistic electronic configuration,

$$
\zeta m \equiv\left(n_{1} l_{1}\right)_{j_{1}}^{w_{1}}\left(n_{2} l_{2}\right)_{j_{2}}^{w_{2}} \cdots\left(n_{\lambda} l_{\lambda}\right)_{j_{\lambda}}^{w_{\lambda}}
$$

with $\sum_{i=1}^{\lambda} w_{i}=Z-\zeta$. As usual, $n_{i}, l_{i}$, and $j_{i}$ are the principal quantum number and the orbital and total angular momenta for the $i$ th relativistic orbital, respectively; $w_{i}$ is the number of electrons occupying the $i$ th orbital; and $\lambda$ is the total number of relativistic orbitals in each configuration. For clarity, we define the occupancy vector $\mathbf{w}_{\zeta m}$ as

$$
\mathbf{w}_{\zeta m} \equiv\left\{w_{1}, w_{2}, \ldots, w_{\lambda}\right\} .
$$

Then the single-electron Dirac equation is solved for each occupied $n l j$ orbital, wherein the local central potential is an analytical parametric one developed for isolated ions [45]. Although the isolated potential is mostly the option used in АВАKO, ions immersed into weakly [46] and strongly coupled plasmas [47] can be also modeled. Moreover, both for isolated and nonisolated situations, corrections for singly and doubly excited configurations can be considered following the method suggested in [48]. Using the built-in model no self-consistent procedure is needed to solve the Dirac equation, which is a great advantage in order to optimize detailed calculations. Furthermore, a relativistic screened hydrogenic model [49] is also available for situations where 
faster calculations are required, e.g., in-line hydrosimulations.

Once the Dirac equation has been solved and the large and small radial components of the one-electron Dirac spinor wave functions as well as the energy eigenvalues are known for the occupied $n l j$ orbitals, then the radial density of bound electrons is calculated and the total energy $E_{\zeta m}$ of the level (configuration) $\zeta m$ is determined following an expression coming from a density-functional theory [50]. On the other hand, since the values of the ionization potentials are critical for a proper determination of the population balance, we use experimental values when they are available. Otherwise, we take them from internet databases [51] or calculations provided by sophisticated codes [52,53].

The ABAKO atomic model assumes the electric dipole approximation to describe the interaction between the atomic system and the electromagnetic field. Also, in the DCA context, a bound-bound transition $\zeta_{i} \rightarrow \zeta j$ with $E_{\zeta i}<E_{\zeta j}$ occurs only if the associated occupancy vectors differ in the excitation of a single electron, i.e.,

$$
\begin{gathered}
\mathbf{w}_{\zeta i} \equiv\left\{w_{1}, \ldots, w_{\eta}, \ldots, w_{\eta^{\prime}}, \ldots, w_{\lambda}\right\}, \\
\mathbf{w}_{\zeta j} \equiv\left\{w_{1}, \ldots, w_{\eta}-1, \ldots, w_{\eta^{\prime}}+1, \ldots, w_{\lambda}\right\} .
\end{gathered}
$$

Then, the absorption oscillator strength $f_{i j}$ is evaluated as follows [54]:

$$
f_{i j}=w_{\eta}\left(1-\frac{w_{\eta}^{\prime}}{2 j_{\eta^{\prime}}+1}\right) f_{\eta \eta^{\prime}},
$$

where $f_{\eta \eta^{\prime}}$ is the monoelectronic oscillator strength for the transition between the relativistic orbitals $\eta \equiv n_{\eta} l_{\eta} j_{\eta}$ and $\eta^{\prime}$ $\equiv n_{\eta^{\prime}} l_{\eta^{\prime}} j_{\eta^{\prime}}$.

The methodology described above allows us to construct a comprehensive set of energy levels-including autoionizing states-and radiative transitions to compute the population distributions. When higher accuracy is required, e.g., for spectroscopic purposes, more detailed atomic structure from databases or computational codes can be used. At present time, the external source often used in ABAKO is FAC [53]. FAC is designed to provide atomic data in DLA approach, but it can also works in a DCA mode by means of configuration averages of detailed levels. A simple computational program has been written to automatically convert the FAC output files into properly formatted ABAKO input files.

\section{Plasma effects}

As the plasma density increases, screening effects due to neighboring electrons and ions begin to modify the energy levels while degeneracy begins to raise the energy of the free electrons. The effect on the ionization potentials of bound states and level populations leads to the phenomenon of pressure ionization. In spite of the importance of pressure ionization to calculate the ionic abundances and level populations, most CR models take into account plasma effects in an approximate way via an effective lowering of the ionization potential or continuum lowering $(\mathrm{CL})$. This is the procedure that we follow in ABAKO, too. Thus, the isolated ion ionization potential $I_{\zeta}$ is lowered a quantity $\Delta I_{\zeta}$ to obtain a noniso- lated value $I_{\zeta}^{\prime}=I_{\zeta}-\Delta I_{\zeta}$. To calculate the correction $\Delta I_{\zeta}$ we apply the formulation due to Stewart and Pyatt [55], but using the particular proposal given in [56],

$$
\Delta I_{\zeta}=\frac{3}{2} \frac{I_{H} a_{0}}{R_{\zeta}}(\zeta+1)\left\{\left[1+\left(\frac{D}{R_{\zeta}}\right)^{3}\right]^{2 / 3}-\left(\frac{D}{R_{\zeta}}\right)^{2}\right\} .
$$

Here, $I_{H}$ is the energy equivalent Rydberg constant, $a_{0}$ is the Bohr radius, $R_{\zeta}=\left[3(\zeta+1) /\left(4 \pi n_{e}\right)\right]^{1 / 3}$ is the ion-sphere radius assuming the plasma composed of ions with charge $\zeta$ only, $D=\left[4 \pi\left(\bar{Z}+\overline{Z^{2}}\right) n_{i o n} / T_{e}\right]^{-1 / 2}$ is the Debye length, $\bar{Z}$ is the plasma average ionization, $\overline{Z^{2}}$ is the second-order moment of the population distribution, $n_{\text {ion }}$ is the total ion density, $n_{e}$ is the free-electron density, and $T_{e}$ is the electron temperature (assuming ion-electron thermalization).

The application of the CL correction brings a significant reduction in the total number of bound states. We perform this process in the following way. Once the lowered ionization potential $I_{\zeta}^{\prime}$ has been calculated, we determine the maximum value $n_{\max }$ reached by the principal quantum number for any electronic configuration $\zeta m$ with energy $E_{\zeta m}<I_{\zeta}^{\prime}$. Then, all the electronic configurations in which there is some occupied relativistic orbital $n l j$ with $n>n_{\max }$ are located and removed from the $\mathrm{CR}$ model. It is interpreted that the monoelectronic orbital $n l j$ is no longer bounded by the potential quantum well. A more detailed discussion about these criteria and others can be found in [56]. Note that when the CL correction is applied the kinetics rate equations must be solved iteratively since the atomic data depend on ionization balance by means of $\bar{Z}$ and $\overline{Z^{2}}$.

\section{B. Collisional-radiative module}

This is the main module in ABAKO. We follow the standard NLTE modeling approach, where an account of the atomic states consistent with $\mathrm{CL}$ is made and the microscopic (radiative and collisional) processes connecting these states are identified. Then, a rate equation system describing the population density of the atomic states is built and solved, giving the population distribution. In the steady-state approximation the following system of rate equations is set up and solved:

$$
\sum_{\zeta^{\prime} j} N_{\zeta^{\prime} j} \mathbb{R}_{\zeta^{\prime} j \rightarrow \zeta i}^{+}-\sum_{\zeta^{\prime} j} N_{\zeta i} \mathbb{R}_{\zeta i \rightarrow \zeta^{\prime} j}^{-}=0,
$$

where $N_{\zeta i}$ is the population density of the atomic level $\zeta i$. The terms $\mathbb{R}_{\zeta^{\prime} j \rightarrow \zeta i}^{+}$and $R_{\zeta^{\prime} j \rightarrow \zeta i}^{-}$take into account all the atomic processes which contribute to populate and depopulate the state $\zeta i$, respectively. In this paper the effects of an external radiation field on the ionization balance will not be considered and the reabsorption effects on the plasma selfemission are addressed following the escape factor approximation. In addition, a Maxwellian energy distribution with a characteristic temperature $T_{e}$ for free electrons is assumed. Table I displays a list with the atomic processes included in $\mathrm{ABAKO}$ and the notation adopted for the corresponding rate coefficients. For the sake of completeness, we can write the total population $\mathrm{R}_{\zeta^{\prime} j \rightarrow \zeta i}^{+}$and depopulation $\mathrm{R}_{\zeta^{\prime} j \rightarrow \zeta i}^{-}$rates in terms of the rate coefficients. Thus, Eq. (6) yields 
TABLE I. List of the atomic processes included in ABAKO and notation for the rate coefficients.

\begin{tabular}{lcccc}
\hline \hline & Direct process & Rate coefficient & Inverse process & Rate coefficient \\
\hline$X_{i}^{+\zeta}+\gamma \Leftarrow X_{j}^{+\zeta}$ & & & Spontaneous decay & $\mathcal{A}_{\zeta j \rightarrow \zeta i}$ \\
$X_{i}^{+\zeta}+e^{-} \Leftrightarrow X_{j}^{+\zeta}+e^{-}$ & Collisional excitation & $n_{e} \mathcal{E}_{\zeta i \rightarrow \zeta j}$ & Collisional de-excitation & $n_{e} \mathcal{D}_{\zeta j \rightarrow \zeta i}$ \\
$X_{i}^{+\zeta}+\gamma \Leftarrow X_{j}^{+\zeta+1}+e^{-}$ & & & Radiative recombination & $n_{e} \mathcal{R}_{\zeta+1 j \rightarrow \zeta i}^{r}$ \\
$X_{i}^{+\zeta}+e^{-} \Leftrightarrow X_{j}^{+\zeta+1}+e^{-}+e^{-}$ & Collisional ionization & $n_{e} \mathcal{I}_{\zeta i \rightarrow \zeta+1 j}$ & Three-body recombination & $n_{e}^{2} \mathcal{R}_{\zeta+1 j \rightarrow \zeta i}^{3}$ \\
$X_{i}^{+\zeta} \Leftrightarrow X_{j}^{+\zeta+1}+e^{-}$ & Autoionization & $\mathcal{A} u_{\zeta i \rightarrow \zeta+1 i}$ & Electron capture & $n_{e} \mathcal{C}_{\zeta+1 j \rightarrow \zeta i}$ \\
\hline \hline
\end{tabular}

$$
\begin{aligned}
& \sum_{j} N_{\zeta-1 j}\left(n_{e} \mathcal{I}_{\zeta-1 j \rightarrow \zeta i}+\mathcal{A} u_{\zeta-1 j \rightarrow \zeta i}\right)+\sum_{\substack{j \\
(j<i)}} N_{\zeta j} n_{e} \mathcal{E}_{\zeta j \rightarrow \zeta i} \\
& \quad+\sum_{j} N_{\zeta j}\left(\mathcal{A}_{\zeta j \rightarrow \zeta i}+n_{e} \mathcal{D}_{\zeta j \rightarrow \zeta i}\right) \\
& \quad+\sum_{j} N_{\zeta+1 j} n_{e}\left(\mathcal{R}_{\zeta+1 j \rightarrow \zeta i}^{r}+n_{e} \mathcal{R}_{\zeta+1 j \rightarrow \zeta i}^{3}+\mathcal{C}_{\zeta+1 j \rightarrow \zeta i}\right) \\
& \quad-N_{\zeta i} \sum_{j} n_{e}\left(\mathcal{R}_{\zeta i \rightarrow \zeta-1 j}^{r}+n_{e} \mathcal{R}_{\zeta i \rightarrow \zeta-1 j}^{3}+\mathcal{C}_{\zeta i \rightarrow \zeta-1 j}\right) \\
& \quad-N_{\zeta i} \sum_{j}\left(\mathcal{A}_{\zeta i \rightarrow \zeta j}+n_{e} \mathcal{D}_{\zeta i \rightarrow \zeta j}\right)-N_{\zeta i} \sum_{j} n_{e} \mathcal{E}_{\zeta i \rightarrow \zeta j} \\
& \quad-N_{(j<i)} \sum_{j}\left(n_{e} \mathcal{I}_{\zeta i \rightarrow \zeta+1 j}+\mathcal{A} u_{\zeta i \rightarrow \zeta+1 j}\right)=0 .
\end{aligned}
$$

Two complementary equations which have to be satisfied together with Eq. (6) are, first, the requirement that the sum of all the partial densities equals the total ion density,

$$
\sum_{\zeta=0}^{Z} \sum_{i=0}^{M_{\zeta}} N_{\zeta i}=N_{i o n}
$$

and, second, the charge neutrality condition in the plasma,

$$
\sum_{\zeta=0}^{Z} \sum_{i=0}^{M_{\zeta}-1} \zeta N_{\zeta i}=n_{e}
$$

where $M_{\zeta}$ is the number of levels in the charge state $\zeta$.

\section{Calculation of the rate coefficients}

Here, we discuss the analytical formulas employed to calculate the rate coefficients of the atomic processes.

(a) Spontaneous decay. The spontaneous emission rate from $\zeta j$ to $\zeta i$ is given by the Einstein coefficient for this process,

$$
\mathcal{A}_{\zeta j \rightarrow \zeta i}=\frac{2 \pi \alpha^{3}}{h I_{H}} E_{i j}^{2} \frac{g_{\zeta i}}{g_{\zeta j}} f_{i j} .
$$

Here, $g_{\zeta i}$ and $g_{\zeta j}$ are the statistical weights of the states, $E_{i j}$ $=E_{\zeta j}-E_{\zeta i}$ is the transition energy, and $f_{i j}$ is the absorption oscillator strength.

(b) Collisional excitation and de-excitation. Let us consider the electron collisional excitation from a state $\zeta i$ to $\zeta j$. To evaluate the collisional excitation cross section we use the formula of Van Regemorter [57],

$$
\sigma_{\zeta i \rightarrow \zeta j}^{e x c}(\varepsilon)=\frac{8 \pi^{2} a_{0}^{2}}{\sqrt{3}}\left(\frac{I_{H}}{E_{i j}}\right)^{2} \frac{g\left(\varepsilon / E_{i j}\right)}{\varepsilon / E_{i j}} f_{i j},
$$

where $\varepsilon$ is the incoming electron energy, $E_{i j}=E_{\zeta j}-E_{\zeta i}$ is the excitation threshold energy, and $g(u)$ is the effective Gaunt factor. We use the Gaunt factor suggested by Mewe [58], i.e., $g(u)=A+(B / u)+\left(C / u^{2}\right)+D \ln u$, where the coefficients $A$, $B, C$, and $D$ can be obtained by fitting to advanced theoretical calculations or experimental data. However, ABAKO follows the general criterion proposed in [58], that is, $A=0.15$ for transitions with $\Delta n=n_{\eta^{\prime}}-n_{\eta}=0$ and $A=0.6$ when $\Delta n$ $\neq 0$, while for the remaining coefficients it is taken as $B$ $=C=0$ and $D=0.28$.

To determine the collisional excitation rate coefficient the cross section is averaged over the free-electron distribution. Assuming a Maxwellian distribution we find

$$
\mathcal{E}_{\zeta i \rightarrow \zeta j}=\frac{16 \sqrt{2} \pi^{2} a_{0}^{2}}{\sqrt{3 \pi m_{e}}} \frac{I_{H}^{2} f_{i j}}{E_{i j} T_{e}^{1 / 2}} \bar{g}\left(E_{i j} / T_{e}\right) e^{-E_{i j} / T_{e}},
$$

where the averaged Gaunt factor is given by $\bar{g}(u)=A+(B u$ $\left.-C u^{2}+D\right) e^{u} E_{1}(u)+C u$, with $E_{1}(u)=\int_{0}^{1}\left(e^{-u / x} / x\right) d x$ being the first-order exponential integral.

The rate coefficient for collisional de-excitation is obtained using the detailed balance principle,

$$
\mathcal{D}_{\zeta j \rightarrow \zeta i}=\frac{g_{\zeta i}}{g_{\zeta j}} e^{E_{i j} / T_{e}} \mathcal{E}_{\zeta i \rightarrow \zeta j} .
$$

On the other hand, it is well known that Eq. (11) is accurate only for transitions within the same shell $n_{\eta}=n_{\eta^{\prime}}$ and its limitations have been clearly shown [59]. Nevertheless, the Van Regemorter formula is still frequently used in atomics kinetics modeling because, first, it is very easy to implement and, second, in the context of large-scale kinetic calculations, it is accurate enough to obtain reasonable estimates of coarse plasma quantities such as average ionization or ionic populations. For those cases, where more accuracy is needed, the Coulomb-Born hydrogenic semiempirical rates by Golden and Sampson [60] might be helpful as an alternative option to the Van Regemorter-Mewe rates.

(c) Collisional ionization and three-body recombination. Another important process is the collisional ionization from a state $\zeta i$ to $\zeta+1 j$. In this case a bound electron of the initial state turns into a free electron, so the occupancy vectors for the initial and final states are

$$
\mathbf{w}_{\zeta i} \equiv\left\{w_{1}, \ldots, w_{\eta}, \ldots, w_{\lambda}\right\},
$$




$$
\mathbf{w}_{\zeta+1 j} \equiv\left\{w_{1}, \ldots, w_{\eta}-1, \ldots, w_{\lambda}\right\} .
$$

To calculate the collisional ionization cross section we use the simple, and yet surprisingly successful, expression of Lotz [61],

$$
\sigma_{\zeta i \rightarrow \zeta+1 j}^{i o n}(\varepsilon)=C \pi a_{0}^{2} I_{H}^{2} w_{\eta} \frac{\ln \left(\varepsilon / E_{i j}\right)}{\varepsilon E_{i j}} .
$$

Here, we take for the constant $C$ the suggested value of 2.77 , $\varepsilon$ is the energy of the incident electron, and now $E_{i j}=I_{\zeta}$ $-E_{\zeta i}+E_{\zeta+1 j}$ is the ionization threshold energy.

Integrating over a Maxwellian distribution, we have the rate coefficient for electron impact ionization

$$
\mathcal{I}_{\zeta i \rightarrow \zeta+1 j}=C \sqrt{\frac{8 \pi}{m_{e}}} a_{0}^{2} I_{H}^{2} \frac{w_{\eta}}{T_{e}^{1 / 2} E_{i j}} E_{1}\left(E_{i j} / T_{e}\right) .
$$

Another option in ABAKO is the slightly modified semiempirical formula of Burgess and Chidichimo [62],

$$
\sigma_{\zeta i \rightarrow \zeta+1 j}^{i o n}(\varepsilon)=C \pi a_{0}^{2} I_{H}^{2} w_{\eta} \frac{\ln \left(\varepsilon / E_{i j}\right)}{\varepsilon E_{i j}} W\left(\varepsilon / E_{i j}\right),
$$

where $C=2.7$ and, for $\varepsilon>E_{i j}$,

$$
W\left(\varepsilon / E_{i j}\right)=\left[\ln \left(\varepsilon / E_{i j}\right)\right]^{\beta E_{i j} / \varepsilon},
$$

with

$$
\beta=\frac{1}{4}\left[\left(\frac{100 \zeta+91}{4 \zeta+3}\right)^{1 / 2}-5\right] .
$$

The only difference between Eqs. (15) and (17) is the factor $W\left(\varepsilon / E_{i j}\right)$, which may have a better representation of the cross-section calculation for near-neutral ions and for electron energies $\varepsilon$ close to the ionization threshold.

Using the Burgess-Chidichimo cross section, the collisional ionization rate results in

$$
\mathcal{I}_{\zeta i \rightarrow \zeta+1 j}=C \sqrt{\frac{8 \pi}{m_{e}}} a_{0}^{2} I_{H}^{2} \frac{w_{\eta}}{T_{e}^{1 / 2} E_{i j}} E_{1}\left(E_{i j} / T_{e}\right) \bar{W},
$$

with $\bar{W}=\left[\ln \left(1+T_{e} / E_{i j}\right)\right]^{\beta /\left(1+T_{e} / E_{i j}\right)}$. In addition to the expressions discussed above, the fits made in $[63,64]$ to CoulombBorn-exchange calculations of ionization scaled cross section of hydrogenlike ions might be considered. The empirical formula proposed in [65] could be another option for ionization from $s, p$, and $d$ states.

The expressions for the collisional ionization cross section and the corresponding rate coefficient discussed here are strictly applicable in a DCA context. In order to perform DLA calculations the cross section should be multiplied by a branching ratio given by $b=g_{\zeta+1 j} / g_{\zeta+1 j}^{\text {tot }}$. Here, $g_{\zeta+1 j}$ now represents the degeneracy of the final well-defined $J$-level $\zeta$ $+1 j$ and $g_{\zeta+1 j}^{t o t}$ is the degeneracy of the configuration associated with $\zeta+1 j$.

For a Maxwellian electron distribution, the three-body recombination rate coefficient is related to the ionization rate coefficient by the detailed balance as

$$
\mathcal{R}_{\zeta+1 j \rightarrow \zeta i}^{3}=\frac{1}{2}\left(\frac{h^{2}}{2 \pi m_{e} T_{e}}\right)^{3 / 2} \frac{g_{\zeta i}}{g_{\zeta+1 j}} e^{E_{i j} / T_{e}} \mathcal{I}_{\zeta i \rightarrow \zeta j} .
$$

(d) Radiative recombination. The starting point to get the radiative recombination rate coefficient from $\zeta+1 j$ to $\zeta i$ is the Milne formula, which relates the cross sections of radiative recombination and photoionization,

$$
\sigma_{\zeta+1 j \rightarrow \zeta i}^{r a d}(\varepsilon)=\frac{\alpha^{2}}{4 I_{H}} \frac{g_{\zeta i}}{g_{\zeta+1 j}} \frac{(h \nu)^{2}}{\varepsilon} \sigma_{\zeta i \rightarrow \zeta+1 j}^{p h o}(\nu),
$$

with $h \nu$ being the energy of the emitted photon. Second, we use the well-known Kramers formula [66] to determine the photoionization cross section,

$$
\sigma_{\zeta i \rightarrow \zeta+1 j}^{p h o}(\nu)=\frac{64 \pi \alpha a_{0}^{2}}{3 \sqrt{3}} \frac{\omega_{\eta} n_{\eta}}{Z_{e f f}^{2}}\left(\frac{E_{i j}}{h \nu}\right)^{3},
$$

where $E_{i j}=I_{\zeta}-E_{\zeta i}+E_{\zeta+1 j}$ is the photoionization threshold energy and $Z_{e f f}=\zeta+1$ is the effective nuclear charge. On the other hand, the energy conservation condition requires $h \nu$ $=E_{i j}+\varepsilon$. Thus, substituting Eq. (23) in Eq. (22), it yields

$$
\sigma_{\zeta+1 j \rightarrow \zeta i}^{r a d}(\varepsilon)=\frac{16 \pi \alpha^{3} a_{0}^{2}}{3 \sqrt{3}} \frac{g_{\zeta i}}{g_{\zeta+1 j}} \frac{w_{\eta} n_{\eta}}{Z_{e f f}^{2}} \frac{E_{i j}^{3}}{I_{H} \varepsilon\left(E_{i j}+\varepsilon\right)} .
$$

Integrating over a Maxwellian distribution, we finally get the expression employed in ABAKO to compute the radiative recombination rate coefficient,

$$
\mathcal{R}_{\zeta+1 j \rightarrow \zeta i}^{r}=\frac{128 \pi^{3 / 2} \alpha^{3} a_{0}^{3}}{3 \sqrt{3} h} \frac{g_{\zeta i}}{g_{\zeta+1 j}} \frac{w_{\eta} n_{\eta}}{Z_{e f f}} \frac{E_{i j}^{3}}{I_{H}^{1 / 2} T_{e}^{3 / 2}} E_{1}\left(E_{i j} / T_{e}\right) e^{E_{i j} / T_{e}} .
$$

In a DLA approach, the radiative recombination cross section is obtained multiplying Eq. (24) by the branching ratio $b=g_{\zeta+1 j} / g_{\zeta+1 j}^{t o t}$, which has exactly the same meaning as was discussed above.

(e) Autoionization and electron capture. The excitation of an inner-subshell electron, or of two or more electrons from any subshells, may lead to states above the ionization limit, i.e., the autoionizing states. The autoionizing states overlap and interact with continuum states in the same ion thus leading to spontaneous autoionization (AU). The inverse process is the electron capture (EC). It differs from radiative recombination in that the kinetic energy of the incoming electron is used to excite another electron in the recombined ion instead of being lost in the emission of a photon. Therefore, the impact electron must have exactly a specific energy to participate in a given electron capture process.

Many papers have highlighted the importance of the AU/EC processes to determine the population balance and radiative properties in plasmas with diverse physical conditions. A few examples are [67-72]. However, AU/EC is hard to model and not all CR models consider an explicit treatment of autoionizing states. The reasons are several. First, the accurate quantum-mechanical evaluation of the autoionization transition probability is quite demanding from a computational point of view [52] and, second, when the autoionizing states are considered, a population transfer between autoionizing and nonautoionizing states can also take place through other collisional and radiative mechanisms. There- 
fore, including the autoionizing states leads to a significant increase in the computation time spent to calculate the atomic processes rate coefficients.

Such quantum-mechanical calculations cannot be afforded in the context of a fast and versatile code. Hence, a simplified model is needed to compute the AU/EC rates. Nevertheless, the problem is that, contrary to what happens with other atomic processes, no analytical formula is known for determining the AU/EC rate coefficients. To overcome this difficulty, we finally opted for a formalism first proposed in [73] and later considered in [74] to obtain an approximate mean value for the electron capture rate. Although it had already been used in the context of a hydrogenic approach [25] ABAKO is a CR model based on analytical expressions that, still working in a DCA scheme, uses this method for an explicit treatment of autoionizing states for performing largescale kinetic calculations.

First we calculate the electron capture rate coefficient from the bound state $\zeta+1 j$ to the autoionizing one $\zeta i$,

$$
\mathcal{C}_{\zeta+1 j \rightarrow \zeta i}=\int_{\Delta E} v \sigma_{\zeta+1 j \rightarrow \zeta i}^{c a p}(\varepsilon) f(\varepsilon) d \varepsilon,
$$

where $v=\sqrt{2 \varepsilon / m_{e}}$ is the free-electron velocity, $\sigma_{\zeta+1 j \rightarrow \zeta i}^{c a p}(\varepsilon)$ is the electron capture cross section, and $f(\varepsilon)$ the free-electron energy distribution function. Since the electron capture is a resonant process the average expressed in Eq. (26) is performed over a narrow interval of width $\Delta E$ around the resonance energy $E_{i j}=E_{\zeta i}-I_{\zeta}-E_{\zeta+1 j}$. Note that $E_{\zeta i}-I_{\zeta}$ is the energy of the autoionizing state with respect to the ionization limit for the ion with charge $\zeta$.

Within the interval $E_{i j} \pm \Delta E / 2$ functions in the integrand can approximately be considered as constants, including the more usual functional forms for the electron distribution, so a good approximation for the integral consists of taking

$$
\mathcal{C}_{\zeta+1 j \rightarrow \zeta i} \approx\left(\frac{2 E_{i j}}{m_{e}}\right)^{1 / 2} \sigma_{\zeta+1 j \rightarrow \zeta i}^{c a p}\left(E_{i j}\right) f\left(E_{i j}\right) \Delta E .
$$

It seems reasonable to think that the captured electron will end up occupying an outer orbital of the ion with charge $\zeta$, because the interaction with continuum states is in fact stronger for such orbitals. Thus, the energy difference between the initial and the final states will be roughly equal to the binding energy for the captured electron, which in turn can be evaluated following a hydrogenic approximation,

$$
E_{i j} \approx \frac{I_{H} Z_{e f f}^{2}}{n^{2}}
$$

with $n$ being the principal quantum number of the referred orbital and $Z_{e f f}=\zeta+1$ being an effective nuclear charge. Also, we can estimate the electron capture width simply by differentiating Eq. (28),

$$
\Delta E \approx \frac{2 I_{H} Z_{e f f}^{2}}{n^{3}} .
$$

Thus, it is considered that free electrons in the interval $E_{i j} \pm \Delta E / 2$ can be captured into all $n l j$ of a given principal quantum number in the sense of an averaged cross section.
Now, the cross section of electron capture from $\zeta+1 j$ to $\zeta i$ is approximated by the cross section of collisional excitation from $\zeta+1 j$ to a particular state $\zeta+1 m$,

$$
\sigma_{\zeta+1 j \rightarrow \zeta i}^{c a p}(\varepsilon) \approx \sigma_{\zeta+1 j \rightarrow \zeta+1 m}^{e x c}(\varepsilon) .
$$

The state $\zeta+1 m$ is called the auxiliary state and it is a bound state of the recombining ion characterized by having the same energy as the autoionizing state $\zeta i$ with respect to the ionization threshold, i.e., $E_{\zeta+1 m}=E_{\zeta i}-I_{\zeta}$.

Thus, we finally obtain for the electron capture rate coefficient the following expression:

$$
\mathcal{C}_{\zeta+1 j \rightarrow \zeta i} \approx\left(\frac{2 E_{i j}}{m_{e}}\right)^{1 / 2} \frac{2 I_{H} Z_{e f f}^{2}}{n^{3}} \sigma_{\zeta+1 j \rightarrow \zeta+1 m}^{e x c}\left(E_{i j}\right) f\left(E_{i j}\right) .
$$

If we use the Van Regemorter formula [Eq. (11)] to calculate the collisional excitation cross section and a Maxwellian distribution is assumed, it leads to

$$
\mathcal{C}_{\zeta+1 j \rightarrow \zeta i}=\frac{32 \pi \sqrt{2 \pi} a_{0}^{2} I_{H}^{2}}{\sqrt{3 m_{e}}} \frac{I_{H} Z_{e f f}^{2}}{n^{3}} f_{j m} \frac{g(1)}{E_{i j} T_{e}^{3 / 2}} e^{-E_{i j} / T_{e}},
$$

where $f_{j m}$ is the absorption oscillator strength associated with the transition $\zeta+1 j \rightarrow \zeta+1 m$.

To determine the autoionization rate coefficient we proceed as in previous sections to use detailed balance,

$$
\mathcal{A} u_{\zeta i \rightarrow \zeta+1 j}=2\left(\frac{2 \pi m_{e}}{h^{2}}\right)^{3 / 2} \frac{g_{\zeta+1 j}}{g_{\zeta i}} T_{e}^{3 / 2} e^{E_{i j} / T_{e}} \mathcal{C}_{\zeta i \rightarrow \zeta+1 j} .
$$

It is important to note the existing relations in ABAKO between the occupancy vectors of the autoionizing state $\zeta i$, the auxiliary $\zeta+1 m$, and the ionized state $\zeta+1 j$. The first two differ in the ionization of one single electron while the last two do it in the excitation of one electron. This means that the autoionization and electron capture processes may cause an electron redistribution besides a different number of bound electrons between the initial and final states. This fact can be represented as

$$
\begin{gathered}
\mathbf{w}_{\zeta i} \equiv\left\{\ldots, w_{\eta}, \ldots, w_{\eta^{\prime}}, \ldots, w_{\delta}, \ldots\right\}, \\
\mathbf{w}_{\zeta+1 m} \equiv\left\{\ldots, w_{\eta}, \ldots, w_{\eta^{\prime}}, \ldots, w_{\delta}-1, \ldots\right\}, \\
\mathbf{w}_{\zeta+1 j} \equiv\left\{\ldots, w_{\eta}+1, \ldots, w_{\eta^{\prime}}-1, \ldots, w_{\delta}-1, \ldots\right\} .
\end{gathered}
$$

The auxiliary state $\zeta+1 m$ in ABAKO is the one over all the bound states of the charge state $\zeta+1$ which has the closest energy to the value $E_{\zeta i}-I_{\zeta}$ and also satisfying relation (34) between the occupancy vectors.

It should be clear that the formalism above represents only a crude approximation for estimating the rate coefficients of electron capture and autoionization and it does not intend to be a way of calculating accurate electron capture cross sections. Its validity must be understood as limited to within the context of large-scale population kinetics calculations.

\section{Radiation transport effects}

Another effect to be considered is the line radiation trapping on population distribution. In the optically thin approxi- 
mation, photons do not interact with the source plasma and therefore radiation-driven atomic processes are neglected. In large size or dense plasmas, however, there may be a significant chance that a photon with energy near that of a resonant bound-bound transition will encounter an ion in the lower level of that transition and undergo absorption before it escapes the plasma. Stimulated emission can also occur if the photon interacts with an ion in the upper level of the resonant transition. Thus, we use the escape factor formalism as an approximate way to account for the bound-bound opacity effects. For a given line transition $\zeta i \leftrightarrow \zeta j$, the escape factor $\Lambda_{j i}$ is introduced as

$$
N_{\zeta j} \mathcal{A}_{\zeta j \rightarrow \zeta i}+\left(N_{\zeta j} \mathcal{B}_{\zeta j \rightarrow \zeta i}^{e}-N_{\zeta i} \mathcal{B}_{\zeta i \rightarrow \zeta j}^{a}\right) J_{i j}=N_{\zeta j} \Lambda_{j i} \mathcal{A}_{\zeta j \rightarrow \zeta i},
$$

where $\mathcal{B}_{\zeta j \rightarrow \zeta i}^{e}$ and $\mathcal{B}_{\zeta i \rightarrow \zeta j}^{a}$ are the Einstein coefficients for stimulated emission and photoabsorption, respectively, and $J_{i j}=J_{j i}$ is the averaged line intensity (assuming that the emission and absorption line profiles are equal).

Escape factors $\Lambda_{j i}$ enter in the calculations in two ways. First, they enter in the atomic physics calculations of excited-state populations. As a result there is an effective reduction in the Einstein spontaneous emission coefficient $\mathcal{A}_{\zeta j \rightarrow \zeta i}$, which is written as $\Lambda_{j i} \mathcal{A}_{\zeta j \rightarrow \zeta i}$. Second, they appear in the determination of the total emergent line intensity. This modification circumvents the need to perform a simultaneous calculation of radiation transport and atomic physics. To compute the escape factors we have adopted the technique described in [75]. Assuming a uniform distribution of emitting atoms and isotropic emission, for the three basic geometries-planar, cylindrical, and spherical-the escape factor is written as

$$
\Lambda_{j i}=\int_{0}^{\infty} \phi_{i j}(\nu) \frac{1}{\tau_{i j}(\nu)} F\left[\tau_{i j}(\nu)\right] d \nu .
$$

Here, $\phi_{i j}(\nu)$ is the line profile. By default ABAKO considers a Voigt profile accounting for natural, collisional, and Doppler broadenings. Stark broadening can be also included as an extra Lorentzian width using the approximate semiempirical formula given in [76]. Other line profile shapes can be used in the escape factor calculations if they are externally provided. Also, $\tau_{i j}(\nu)=\kappa_{\zeta i \rightarrow \zeta j}(\nu) L$ is the optical depth, where in turn $\kappa_{\zeta i \rightarrow \zeta j}(\nu)$ is the line absorption coefficient and $L$ denotes the characteristic plasma dimension, i.e., slab width, cylinder, or sphere radius. Finally, $F\left[\tau_{i j}\right]$ is a functional of the optical depth whose particular form depends on the considered geometry. Thus, for the slab it is obtained that [77]

$$
F(\tau)=\frac{1}{2}-E_{3}(\tau),
$$

with $E_{3}(\tau)$ being the third-order exponential integral, for the spherical case it takes the form [75]

$$
F(\tau)=\frac{3}{4}\left\{1-\frac{1}{\tau^{2}}\left[\frac{1}{2}-\left(\tau+\frac{1}{2}\right) e^{-2 \tau}\right]\right\},
$$

and for the cylindrical geometry $F(\tau)$ is computed by interpolation over a numerically defined function as described in [78]. Unlike other widely used methods for evaluating the escape factor, such as [38-40], the formalism used in ABAKO has the advantage that details about geometry are treated in an exact way. Moreover, according to Eq. (36), the last integral that needs to be solved is a frequency integral over the line profile. This fact facilitates the use of other line profile functions, such as those provided by elaborate Stark calculations [75].

For each line transition, the escape factor $\Lambda_{j i}$ depends implicitly on the populations of the lower, $N_{\zeta i}$, and upper, $N_{\zeta j}$, levels since, according to Eq. (36), they are required to compute the absorption coefficient $\kappa_{\zeta i \rightarrow \zeta j}(\nu)$. Hence, in the case of optically thick plasmas, the system of rate equations must be solved iteratively until convergence is achieved within a prescribed tolerance.

\section{Solution of the linear system of rate equations}

When the electron density is taken as an input parameter, the set of rate equations [Eq. (6)] constitutes a linear system of $M$ equations for the level populations, where $M$ is the total number of levels included in the model. However, the atomic processes usually connect only levels that belong either to the same charge state or to adjacent ones, which leads to a sparse linear system. Thus, in order to keep memory requirements to a minimum, ABAKO uses sparse techniques to store and operate on only the nonzero matrix elements. Additionally, in a population kinetics problem the number of levels can easily reach the order of $10^{4}-10^{5}$; hence, ABAKO uses an iterative method [79] to perform the matrix inversion, because they typically compute the solution significantly faster than a direct (standard) method.

\section{Radiative properties module}

ABAKO comprises a third module, called RAPCAL, which is devoted to determine plasma radiative properties. A standard formulation is followed to perform such calculations. Full details about RAPCAL along with a collection of representative results-including spectrally resolved and mean opacities and emissivities, radiative power losses and transmission spectrum for carbon, aluminum, krypton, and xenon plasmas for LTE and NLTE conditions-have been published in $[80,81]$. Also, a study about the influence of the atomic description and configuration interaction effects on CR calculations and radiative properties of low ionized carbon plasmas was performed in [82]. Hence, no additional comments about this third module will be reported here.

\section{RESULTS}

It is impossible to discuss here all the computational experiments and cases analyzed during the development of ABAKO and previous related work $[77,83,84]$. Therefore, we will only present a set of representative results that allow us to show the capabilities of the model by means of comparisons with other codes and available experimental data. In order to provide a full test bench for the validation of ABAKO, the calculations include plasmas of low-to-high $Z$ elements and cover different ranges of temperatures and densities. We will discuss three different kinds of results. The 
first one consists of an internal consistency analysis in order to check the limits of low and high electron densities. Second, we compare the ABAKO results with other CR codes developed by diverse research groups in the atomic plasma physics community. Such comparisons mainly come from computational tests proposed in the last editions of NLTE workshops [42-44]. Third, ABAKO is used to simulate the ionization balance of several benchmark experiments of xenon and gold plasmas and, finally, the usefulness of ABAKO as a tool for plasma spectroscopic diagnostics is demonstrated by the analysis of the $K$-shell emission spectra originated from laser-produced dense aluminum plasmas obtained at the Laboratoire pour l'Utilisation des Lasers Intenses (LULI) as well as in the implosion of argon-doped deuterium-filled capsules used in inertial confinement fusion (ICF) direct-drive experiments performed at OMEGA.

Nevertheless, before discussing the results, the set of electronic configurations that must be included in a particular kinetic calculation should be addressed. Level populations and radiative properties depend strongly on the atomic configurations included in the calculations $[10,20,85]$. In this connection, the overall analysis of the large number of cases studied during the ABAKO development has led us to consider a complete enough set of configurations, which allows us to obtain reasonable estimates of the ionization balance. Table II shows the list of configurations by isoelectronic sequences from $\mathrm{H}$-like to $\mathrm{Kr}$-like. Especially for ions with a high number of bound electrons and with the unique purpose of having a tractable number of configurations, sometimes the angular momentum $l$ is limited up to a maximum value $l_{\max }$ $<n-1$. Such a set of configurations is used by default in ABAKO for any calculation, regardless of the atomic number $Z$ or the plasma conditions. The table suggests some qualitative rules to build the excited configurations from the lowlying ones. The list of selected configurations has been shown to be robust for atomic kinetic modeling since the addition of more configurations produced hardly appreciable changes in the population distributions, at least in all the cases we have studied.

\section{A. Internal consistency: Low- and high-density limits}

It is well known that the CR steady-state model covers the situations of CE and LTE in the low and high electron density regimes, respectively, but existing an intermediate region in which $\mathrm{CR}$ model gives specific results, i.e., the genuine NLTE region [86]. Thus, as first step to validate ABAKO results, we accomplished an internal study of numerical consistency to prove that ABAKO effectively converges to the results obtained with the $\mathrm{CE}$ model for low electron densities and to the ones given by the Saha-Boltzmann equations in the high-density regime. Systematic calculations for carbon, aluminum, argon, germanium, and gold confirmed that ABAKO exhibits the expected behavior. An example is given in Fig. 1 in which we plot the average ionization of a carbon plasma at $T_{e}=10 \mathrm{eV}$ over a wide range of electron densities. Below $10^{14} \mathrm{~cm}^{-3}$ our model converges to the $\mathrm{CE}$ result showing its characteristic density-independent behavior. On the other side, approximately above $2 \times 10^{19} \mathrm{~cm}^{-3}$, ABAKO
TABLE II. List of electronic configurations for isoelectronic sequences from $\mathrm{H}$-like to $\mathrm{Kr}$-like included by default in the ABAKO kinetics calculations. We specify the configurations using complexes defined according to the occupation numbers of the $n$ shells. Thus, $\left(n_{1}\right)^{w_{1}}\left(n_{2}\right)^{w_{2}} \cdots$ denotes the totality of relativistic configurations with $w_{1}$ electrons in the shell $n=n_{1}, w_{2}$ in $n=n_{3}$, etc.

\begin{tabular}{|c|c|}
\hline Ion & Electronic configurations \\
\hline H-like & $n l, n \leq 10$ \\
\hline He-like & (1) $n l, n \leq 10 ;(2) n l, n \leq 6$; (3) $n l, n \leq 6$ \\
\hline Li-like & $n l, n \leq 10 ;(1)(2) n l, n \leq 6 ;(1)(3) n l, n \leq 6$ \\
\hline Be-like & (2) $n l, n \leq 10 ;(3) n l, n \leq 6$; (1)(2) ${ }^{2} n l, n \leq 6$ \\
\hline B-like & $(2)^{2} n l, n \leq 10 ;(2)(3) n l, n \leq 6 ;(1)(2)^{3} n l, n \leq 6$ \\
\hline C-like & $(2)^{3} n l, n \leq 10 ;(2)^{2}(3) n l, n \leq 6$ \\
\hline N-like & $(2)^{4} n l, n \leq 10 ;(2)^{3}(3) n l, n \leq 6$ \\
\hline O-like & $(2)^{5} n l, n \leq 10 ;(2)^{4}(3) n l, n \leq 6$ \\
\hline F-like & $(2)^{6} n l, n \leq 10 ;(2)^{5}(3) n l, n \leq 6$ \\
\hline Ne-like & $(2)^{7} n l, n \leq 10 ;(2)^{6}(3) n l, n \leq 6$ \\
\hline Na-like & $n l, n \leq 10 ;(2)^{7}(3) n l, n \leq 6$ \\
\hline Mg-like & (3) $n l, n \leq 10 ;(2)^{7}(3)^{2} n l, n \leq 6$ \\
\hline Al-like & $(3)^{2} n l, n \leq 10 ;(2)^{7}(3)^{3} n l, n \leq 6$ \\
\hline Si-like & $(3)^{3} n l, n \leq 10 ;(2)^{7}(3)^{4} n l, n \leq 6$ \\
\hline P-like & $(3)^{4} n l, n \leq 10 ;(2)^{7}(3)^{5} n l, n \leq 6$ \\
\hline S-like & $(3)^{5} n l, n \leq 10 ;(2)^{7}(3)^{6} n l, n \leq 6$ \\
\hline Cl-like & $(3)^{6} n l, n \leq 10 ;(2)^{7}(3)^{7} n l, n \leq 6$ \\
\hline Ar-like & $(3)^{7} n l, n \leq 8 ;(3)^{6}(4)^{2} ;(2)^{7}(3)^{8} n l, n \leq 6$ \\
\hline K-like & $(3)^{8} n l, n \leq 8 ;(3)^{7}(4) n l, n \leq 6$ \\
\hline Ca-like & $(3)^{9} n l, n \leq 8 ;(3)^{8}(4) n l, n \leq 6$ \\
\hline Sc-like & $(3)^{10} n l, n \leq 8 ;(3)^{9}(4) n l, n \leq 6$ \\
\hline Ti-like & $(3)^{11} n l, n \leq 8 ;(3)^{10}(4) n l, n \leq 6$ \\
\hline V-like & $(3)^{12} n l, n \leq 8 ;(3)^{11}(4) n l, n \leq 6$ \\
\hline Cr-like & $(3)^{13} n l, n \leq 8 ;(3)^{12}(4) n l, n \leq 6$ \\
\hline Mn-like & $(3)^{14} n l, n \leq 8 ;(3)^{13}(4) n l, n \leq 6$ \\
\hline Fe-like & $(3)^{15} n l, n \leq 8 ;(3)^{14}(4) n l, n \leq 6$ \\
\hline Co-like & $(3)^{16} n l, n \leq 8 ;(3)^{15}(4) n l, n \leq 6$ \\
\hline Ni-like & $(3)^{17} n l, n \leq 8 ;(3)^{16}(4) n l, n \leq 6$ \\
\hline $\mathrm{Cu}$-like & $n l, n \leq 8 ;(3)^{17}(4) n l, n \leq 6$ \\
\hline Zn-like & (4) $n l, n \leq 8 ;(5)^{2} ;(5)(6) ;(6)^{2} ;(3)^{17}(4)^{2} n l, n \leq 5$ \\
\hline Ga-like & $\begin{array}{c}(4)^{2} n l, n \leq 8 ;(4)(5)^{2} ;(4)(5)(6) ;(4)(6)^{2} \\
3 d^{9}(4)^{3} n l, n \leq 5\end{array}$ \\
\hline Ge-like & $\begin{aligned}(4)^{3} n l, n \leq 8 ; & (4)^{2}(5)^{2} ;(4)^{2}(5)(6) ;(4)^{2}(6)^{2} \\
& 3 d^{9}(4)^{4} n l, n \leq 5\end{aligned}$ \\
\hline As-like & $(4)^{4} n l, n \leq 8 ; 3 d^{9}(4)^{5} n l, n \leq 5$ \\
\hline Se-like & $(4)^{5} n l, n \leq 8 ; 3 d^{9}(4)^{6} n l, n \leq 5$ \\
\hline Br-like & $(4)^{6} n l, n \leq 8 ; 3 d^{9}(4)^{7} n l, n \leq 5$ \\
\hline Kr-like & $(4)^{7} n l, n \leq 8 ; 3 d^{9}(4)^{8} n l, n \leq 5$ \\
\hline
\end{tabular}

satisfactorily reproduces the curve from Saha-Boltzmann. In the intermediate region, $\sim 10^{14}-10^{19} \mathrm{~cm}^{-3}$, a NLTE regime is reached and the CR model predicts an ionization balance different from CE or LTE. As the electron density increases, the collisional excitation becomes more important thus populating the excited states which, in turn, can undergo an electron impact ionization more easily. This leads to an increas- 


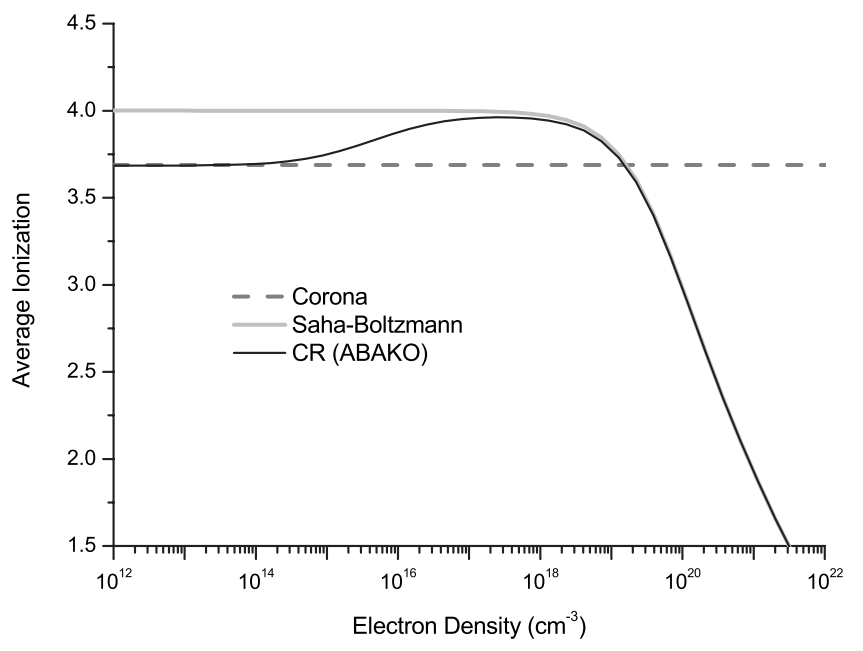

FIG. 1. Average ionization of a carbon plasma at $T_{e}=10 \mathrm{eV}$ over a wide range of electron densities. As it is expected the CR model tends asymptotically to the results obtained with the $\mathrm{CE}$ and Saha-Boltzmann equations in the low and high electron density regimes, respectively.

ing average ionization. Nevertheless, when the electron density is high enough, the three-body recombination process dominates and $\bar{Z}$ starts to drop. These mechanisms allow us to explain the observed bump in the average ionization curve predicted by the CR model.

Since ABAKO includes autoionizing states explicitly, in order to achieve the convergence between coronal and CR results, we need to take into account in the $\mathrm{CE}$ equations the possible increase in the total ionization rate due to the twostep excitation-autoionization mechanism. In this respect, to compute the total ionization rate out of ground state, $\zeta 0$, an extra term must be added in which the collisional excitation rate coefficient $\mathcal{E}_{\zeta 0 \rightarrow \zeta j^{\star}}$ appears multiplied by the branching ratio

$$
b_{\zeta j^{\star}}=\frac{\sum_{j} \mathcal{A} u_{\zeta j^{\star} \rightarrow \zeta+1 j}}{\sum_{j} \mathcal{A} u_{\zeta j^{\star} \rightarrow \zeta+1 j}+\sum_{j} \mathcal{A}_{\zeta j^{\star} \rightarrow j}},
$$

with $\zeta j^{\star}$ denoting an autoionizing state, which measures the probability of autoionization versus radiative stabilization. Analogously, an additional term is included in the total twobody recombination rate, wherein the electronic capture rate coefficient $\mathcal{C}_{\zeta 0 \rightarrow \zeta-1 j^{\star}}$ appears multiplied by the branching ratio $b_{\zeta j^{\star}}^{\prime}=1-b_{\zeta j^{\star}}$.

Additionally, the asymptotic convergence of ABAKO to the CE and LTE results in the opposite limits of electron densities was used in [87] to establish a criterion for discerning coronal, LTE, and NLTE regimes on the electron temperature-density plane of optically thin carbon plasmas. The influence of excited states, individual atomic processes, and CL on the ionization balance was also studied. Further details related to the internal consistency of ABAKO can be found in [77].

\section{B. Comparison with other codes}

Large and exhaustive comparisons were made between the ABAKO results and those provided by other CR models in the plasma kinetics community. The comparisons rely on the analysis of a series of computational tests proposed in the last three editions of the Non-LTE Code Comparison Workshops [42-44]. The results of this analysis were critical for developing and improving the current version of ABAKO. We carried out many calculations for carbon, aluminum, argon, xenon, and gold for a wide range of densities and temperatures. From a general point of view, for the most of quantities analyzed - such as average ionization, charge state distribution, level population distribution, total and fractional (due to each particular atomic process) ionization and recombination rates, and radiative power losses-ABAKO gives good results compared with more sophisticated models. Nevertheless, code identities must be preserved and therefore the referred analysis will not be discussed in this paper. Here, we will restrict our considerations to the analysis of some results of carbon plasmas originally presented in the workshops, which were later on extended and published elsewhere by their corresponding contributors.

In NLTE-4 a set of carbon cases were chosen as a test problem that could be computed in detail using a complete generated model. The range of temperatures (3-10 eV) and densities $\left(10^{13}-10^{19} \mathrm{~cm}^{-3}\right)$ to be studied were previously chosen to ensure genuine NLTE physical conditions. For these carbon test cases the group from Los Alamos National Laboratory (LANL) provided a benchmark atomic model to establish a comparison framework for different codes. Their results have been published also including a more exhaustive exploration of power losses and sample spectra obtained for a variety of plasma conditions [88]. These calculations were obtained using the ATOMIC code. The atomic data were generated in both the configuration average and fine-structure approximations, including intermediate-coupling and configuration-interaction effects. Collisional excitation and ionization, photoionization, and autoionization cross sections were obtained following quantum-mechanical procedures. Thus, in Table III we compare the average ionization for carbon plasmas calculated using ATOMIC, both under DCA and DLA approaches, with those provided by ABAKO. With ABAKO-DCA we denote the results obtained using the built-in DCA atomic structure model based on analytical potentials. Also, in order to show the ABAKO capability for working with DLA external data, we have included the calculations performed with an atomic structure provided by FAC, i.e., ABAKO-DLA. In addition, we reproduce average ionizations determined by Poirier and de Gaufridy de Dortan [89]. For this last case, energy levels and cross sections for different atomic processes were computed using HULLAC. This code includes a fully relativistic formalism based on a sophisticated parametric potential, the accounting for interaction between configurations, an efficient description of continuum wave functions using the phase-amplitude method, and the fast computation of collisional cross sections using the factorization method.

As we can observe, our results are satisfactory. The mean relative errors between ABAKO and ATOMIC for the cases dis- 
TABLE III. Average ionization of the NLTE-4 carbon test case. Results from the LANL code ATOMIC [88], a HULLAC-based CR model [89], and ABAKO are shown for comparison. DCA and DLA calculations are displayed.

\begin{tabular}{|c|c|c|c|c|c|c|c|}
\hline \multirow{2}{*}{$\begin{array}{c}T_{e} \\
(\mathrm{eV})\end{array}$} & \multirow{2}{*}{$\begin{array}{c}n_{e} \\
\left(\mathrm{~cm}^{-3}\right)\end{array}$} & \multicolumn{2}{|c|}{ Colgan et al. } & \multicolumn{2}{|c|}{ Poirier and de Gaufridy de Dortan } & \multicolumn{2}{|c|}{ АВАKO } \\
\hline & & DCA & DLA & DCA & DLA & DCA & DLA \\
\hline \multirow[t]{4}{*}{3} & $10^{13}$ & 1.486 & 1.730 & 1.999 & 1.902 & 1.605 & 1.619 \\
\hline & $10^{15}$ & 1.895 & 1.923 & 2.166 & 2.037 & 1.786 & 1.852 \\
\hline & $10^{17}$ & 1.948 & 1.952 & 2.501 & 1.991 & 1.917 & 1.913 \\
\hline & $10^{19}$ & 0.959 & 1.004 & 1.980 & 1.179 & 1.142 & 0.932 \\
\hline \multirow[t]{4}{*}{5} & $10^{13}$ & 2.055 & 2.165 & & & 2.057 & 2.135 \\
\hline & $10^{15}$ & 2.592 & 2.514 & & & 2.309 & 2.276 \\
\hline & $10^{17}$ & 2.979 & 2.976 & & & 2.948 & 2.878 \\
\hline & $10^{19}$ & 2.104 & 2.076 & & & 2.182 & 2.084 \\
\hline \multirow[t]{4}{*}{7} & $10^{13}$ & 2.729 & 2.887 & & & 2.722 & 2.873 \\
\hline & $10^{15}$ & 3.189 & 3.185 & & & 3.004 & 2.977 \\
\hline & $10^{17}$ & 3.718 & 3.729 & & & 3.529 & 3.545 \\
\hline & $10^{19}$ & 2.993 & 2.987 & & & 3.001 & 2.998 \\
\hline \multirow[t]{4}{*}{10} & $10^{13}$ & 3.701 & 3.723 & 3.739 & 3.746 & 3.684 & 3.705 \\
\hline & $10^{15}$ & 3.856 & 3.862 & 3.828 & 3.828 & 3.747 & 3.745 \\
\hline & $10^{17}$ & 3.978 & 3.979 & 3.976 & 3.976 & 3.955 & 3.956 \\
\hline & $10^{19}$ & 3.785 & 3.786 & 3.835 & 3.833 & 3.776 & 3.787 \\
\hline
\end{tabular}

played are $4.46 \%$ for DCA and $3.14 \%$ for DLA. These represent small differences with respect to the typical discrepancies between models observed in the NLTE workshops. Since the analytical potentials used in the ABAKO atomic module avoid the iterative procedures of the self-consistent calculations, the collection of DCA results indicates that ABAKO-DCA allows us to perform reasonable and fast estimations of plasma average ionization and ion populations. Moreover, the small discrepancies found for the DLA calculations give a valuable proof to validate the use of detailed atomic data provided by FAC into the ABAKO CR module which, by using analytical expressions to calculate the rate coefficients, leads to a substantial reduction in the computation time compared with full-DLA kinetics calculations.

If we make a case-by-case analysis, we find a better agreement between codes for the higher values of temperature and density, clearly due to the approaching to LTE conditions. Nevertheless, at lower densities, where autoionization and electron capture dominate, the differences are greater. Particularly, at $T_{e}=3 \mathrm{eV}$, the DCA calculations performed by Poirier and de Gaufridy de Dortan present large deviations with respect to ATOMIC or ABAKO. We consider that this point requires further discussion. Poirier and de Gaufridy de Dortan attributed the big differences between their DCA and DLA results to the two-step autoionization mechanism $1 s^{2} 2 p^{3} 3 d \rightarrow 1 s^{2} 2 s 2 p 3 s \rightarrow 1 s^{2} 2 s^{2}$, which is not allowed in the detailed calculation, but however it is in the DCA model also showing large autoionization rates. Poirier and de Gaufridy de Dortan argued that Colgan et al. did not notice such a big discrepancy between their DCA and DLA calculations because the configuration $1 s^{2} 2 p^{3} 3 d$ was not accounted for in their calculation. However, our calculations are in a clear disagreement with the conclusion obtained by Poirier and de Gaufridy de Dortan. First, the $1 s^{2} 2 p^{3} 3 d$ configuration is included by default in both DCA- and DLAABAKO calculations and, as it is shown in Table III, we do not obtain big differences between DCA and DLA calculations and a good agreement is found with the corresponding ATOMIC results. Moreover, we removed the $1 s^{2} 2 p^{3} 3 d$ configuration from the $\mathrm{CR}$ model and it made little difference from the calculations including it. Furthermore, we used the ABAKO built-in atomic model for a deeper analysis of the configurations involved in the cascade autoionization mentioned by Poirier and de Gaufridy de Dortan and we found that the energy of the $1 s^{2} 2 s 2 p 3 s$ configuration lie below the B-like C first ionization potential and therefore it does not autoionize. Since the ABAKO atomic model is based on a simple analytical potential, we tried to confirm this fact by analyzing a more accurate energy-level structure provided by other codes. Thus, we used the DCA mode in FAC and the COWAN code [52] - accessible through the LANL website [52] — and we checked that both lead to similar nonautoionizing energies for the $1 s^{2} 2 s 2 p 3 s$ configuration. Therefore, contrary to what Poirier and de Gaufridy de Dortan concluded, the cascade autoionization $1 s^{2} 2 p^{3} 3 d \rightarrow 1 s^{2} 2 s 2 p 3 s$ $\rightarrow 1 s^{2} 2 s^{2}$ is not allowed in either DCA or DLA approach. A plausible explanation for the differences seen by Poirier and de Gaufridy de Dortan between their DCA and DLA results 


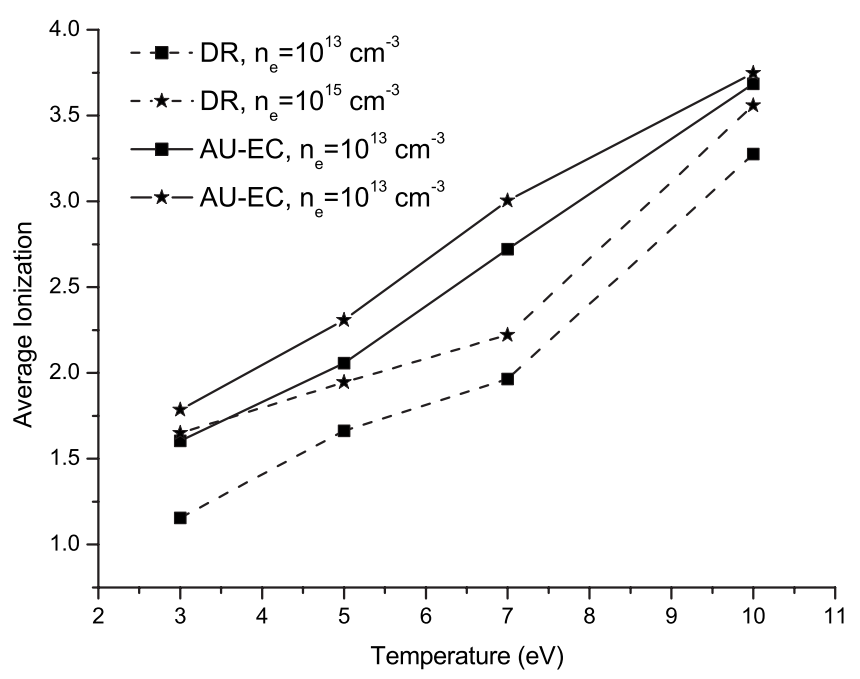

FIG. 2. Average ionization of a carbon plasma at several temperatures and densities. In order to stress the role of autoionizing states, we used ABAKO to compare different calculations: DR does not include autoionizing states explicitly, instead the Burgess-Merts formalism is used to carry out a direct evaluation of the dielectronic recombination rate; AU-EC denotes here the same ABAKO-DCA results shown in Table III, which include autoionizing states explicitly and the rate coefficients of autoionization and electron capture computed as described in the previous section.

may be that the HULLAC level energies were somewhat inaccurate for the $1 s^{2} 2 s 2 p 3 s$ configuration and the Poirier method of taking configuration average (by statistically averaging the fine-structure levels) then resulted in the $1 s^{2} 2 s 2 p 3 s$ configuration being above the ionization limit and large spurious autoionization rates for the $1 s^{2} 2 p^{3} 3 d$ $\rightarrow 1 s^{2} 2 s 2 p 3 s$ and $1 s^{2} 2 s 2 p 3 s \rightarrow 1 s^{2} 2 s^{2}$ transitions.

On the other hand, as was discussed in Sec. II B, an explicit treatment of autoionizing states entails a considerable additional computational effort to determine the rate coefficients, particularly when a full quantum-mechanical calculation is performed. An alternative sometimes used to avoid an explicit treatment of autoionizing states consists of using one of the available analytical approximations for a direct evaluation of the dielectronic recombination (DR) rate coefficient, e.g., the Burgess-Merts formalism [90,91]. However, we found that for low and intermediate electron densities such an approach produces systematically lower average ionizations than those predicted by CR models including explicit and quantum-mechanical rates of the AU/EC processes. To illustrate this behavior, we simply repeated the carbon test case for the two lowest densities, where the AU/EC pair controls the ionization balance, but using the Burgess-Merts formalism to calculate the DR rate coefficient. The results are plotted in Fig. 2. As we can see, the only consideration of DR rates leads to incorrect lower values of average ionization compared to the calculations including the AU/EC pair, with deviations between $5 \%\left(T_{e}=10 \mathrm{eV}, n_{e}=10^{15} \mathrm{~cm}^{-3}\right)$ and $30 \%\left(T_{e}=3 \mathrm{eV}, n_{e}=10^{13} \mathrm{~cm}^{-3}\right)$. Therefore, taking into account the extra computational effort of performing a rigorous evaluation of the AU/EC rates, but noting the importance of such atomic processes for an adequate modeling of the population kinetics, the example of Fig. 2 gives a proof of

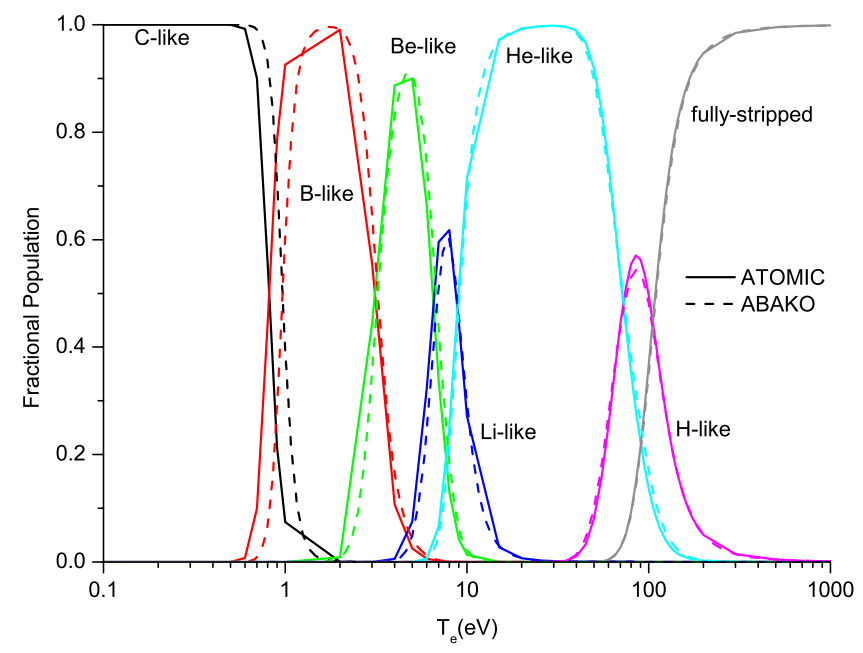

FIG. 3. (Color online) Fractional populations of carbon ion stages calculated with ATOMIC [88] and ABAKO over a wide range of temperatures for a density $n_{e}=10^{13} \mathrm{~cm}^{-3}$.

the utility of the simple approach implemented in ABAKO to calculate the AU/EC rates.

Beyond the analysis of simple global plasma parameters, such as the average ionization, ABAKO can be useful to determine reasonable estimations of ion parameters, such as charge state distribution and total and fractional recombination and ionization rates. To give an example, Fig. 3 shows charge state DCA calculations of a carbon plasma at $n_{e}$ $=10^{13} \mathrm{~cm}^{-3}$ over a wide range of temperatures, i.e., $T_{e}$ $=0.1-1000 \mathrm{eV}$. The ABAKO calculations were performed defining a grid of a hundred logarithmically spaced temperature points and they took about 20 min of computation time on an ordinary PC. Although the population kinetics of such lowdensity plasmas is more difficult to simulate due to the prevailing role of the autoionization and electron capture processes, a very good agreement between the ATOMIC and $A B A K O$ results can be observed over almost the entire range of temperatures. Only for $T_{e}<3 \mathrm{eV}$ some discrepancies are found, which is not surprising because the energy-level structure determined using the analytical potential implemented in ABAKO presents some known inaccuracies for near-neutral species.

\section{Comparison with experimental data}

In addition, ABAKO results have been tested with experimental data involving complex ions from high- $Z$ plasmas.

TABLE IV. Average ionization for the Au experiment performed on the LLNL EBIT-II [92] and calculations obtained by different models assuming $n_{e}=10^{12} \mathrm{~cm}^{-3}$ and $T_{e}=2500 \mathrm{eV}$.

\begin{tabular}{lc}
\hline \hline Source & $\bar{Z}$ \\
\hline Experiment [92] & $46.8 \pm 0.75$ \\
ABAKO & 47.2 \\
AVERROES [93] & 46.4 \\
FLYCHK [25] & 48.5 \\
MIST [92] & 42.7 \\
RIGEL/MCXSN [92] & 49.5 \\
\hline \hline
\end{tabular}




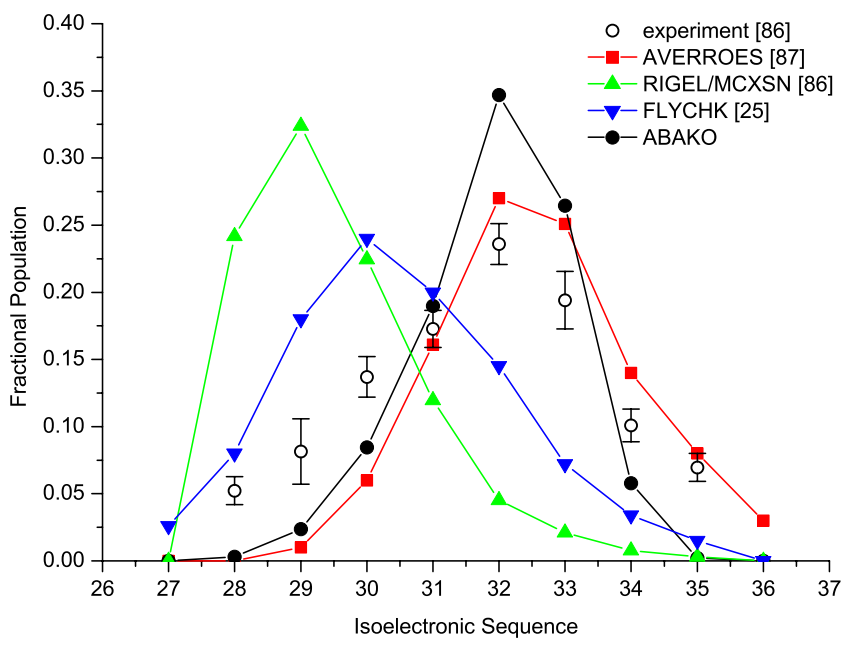

FIG. 4. (Color online) Comparison of charge state distributions simulated by different models and the measurements for the $\mathrm{Au}$ experiment with $n_{e}=10^{12} \mathrm{~cm}^{-3}$ and $T_{e}=2500 \mathrm{eV}$ performed at LLNL EBIT-II.

Calculations were performed to simulate several wellcharacterized experiments of xenon and gold, which cover different electron density and opacity effect domains. The first example turns on the measurement of the charge state distribution of a highly ionized gold plasma in coronal equilibrium [92]. The experiment utilized the electron beam ion trap EBIT-II at the Lawrence Livermore National Laboratory (LLNL) to create a plasma with a Maxwellian temperature of $T_{e}=2.5 \mathrm{keV}$ and $n_{e} \approx 10^{12} \mathrm{~cm}^{-3}$. Such low-density laboratory plasmas offer the advantage of involving fewer active processes (no photoionization and no line absorption) and one can invoke purely optically thin calculations to interpret them. In Table IV we compare the experimental average ionization with the one obtained by ABAKO as well as other published results from different CR models. As we can see only ABAKO and AVERROES predict a value which agrees with the experiment. In Fig. 4 we plot the comparison for the charge state distribution. The distributions calculated by ABAKO and AVERROES are those closest to the experiment, both peaking at the experimental most populated ion. Other calculations are shifted two or three charge states higher. Also, we observe that AVERROES predictions are slightly better than those obtained by ABAKO. This is something that can be expected because AVERROES is a sophisticated superconfiguration-based code in which the radiative and collisional atomic processes rates are calculated by means of quantum-mechanical methods. Nevertheless, according to
[93], the AVERROES version used for the analysis of this experiment includes a calculation procedure which is quite demanding in terms of computation time and memory and is not intended to provide results on a large scale. On the contrary, no particular implementation was required in the ABAKO calculation, which included approximately 42000 relativistic electron configurations from $\mathrm{Kr}$ - to $\mathrm{Ni}$-like $\mathrm{Au}$, taking a few hours of computing time on an ordinary machine.

The interpretation made in [93] of this experiment underlines the strong importance of highly excited configurations which, through the many autoionization and capture channels they open, cannot be forgotten or simply poorly described in a coronal calculation. The analysis using ABAKO leads to the same conclusion. Thus, in Table $\mathrm{V}$ we give the total ionization rate out of ion As-like Au, weighted by the fractional populations of the initial states and summed over all final states, and the corresponding contribution of electron collisional ionization, photoionization, and autoionization, respectively. The total recombination rate out of this ion stage with the corresponding contribution of three-body recombination, radiative recombination, and dielectronic capture has been included, too. The results obtained with AVERROES are also displayed for comparison. As we can see, the strongly prevailing aspect of the AU/EC processes is clearly demonstrated. Additionally, the similarity that we have found between the ABAKO and AVERROES results shows the utility of the procedure implemented in ABAKO for calculating the $\mathrm{AU} / \mathrm{EC}$ rate coefficients.

A second case was an experiment conducted on the NOVA laser facility at LLNL to create a highly ionized Au plasma [94]. The measurements were $n_{e}=6 \times 10^{20} \mathrm{~cm}^{-3} \pm 20 \%, \quad T_{e}=2200 \mathrm{eV} \pm 10 \%, \quad$ and $\bar{Z}$ $=49.3 \pm 1 \%$. Table VI shows a comparison between the experimental $\bar{Z}$ and the calculations obtained by different models. An excellent agreement between ABAKO and the experiment is found. Anyway, for this case predictions are better than for the low-density one, because of the higher density that leads to a plasma state closer to LTE. In Fig. 5 we plot the charge state distribution obtained with the superconfiguation-based code RIGEL, ABAKO, and the measured one. The results show a good agreement with the experiment, although the curve provided by RIGEL is slightly better than the ABAKO result. This is basically due to that the superconfiguration approach works particularly well for high densities, while for the previous low-density Au plasma, the RIGEL results were far from the experiment. On the other hand, in this case opacity effects have been included in the ABAKO calculations assuming a uniform plasma and using

TABLE V. Total ionization and recombination rates out of As-like Au and the fractional contribution of the atomic processes for the LLNL EBIT-II Au plasma. Results from ABAKO and AVERROES are displayed for comparison.

\begin{tabular}{lcccccccc}
\hline \hline & $\begin{array}{c}\text { Tot. ion. } \\
\left(\mathrm{s}^{-1}\right)\end{array}$ & Coll. & Phot. & Auto. & $\begin{array}{c}\text { Tot. rec. } \\
\left(\mathrm{s}^{-1}\right)\end{array}$ & Three-bo. & Rad. & Cap. \\
\hline ABAKO & 166.50 & 0.157 & 0.000 & 0.843 & 82.90 & 0.000 & 0.018 & 0.982 \\
AVERROES & 256.16 & 0.123 & 0.000 & 0.876 & 244.91 & 0.000 & 0.024 & 0.976 \\
\hline \hline
\end{tabular}


the escape factor formalism described in Sec. II B. A Voigt shape taking into account natural and Doppler broadenings was considered for line profile. According to the experiment an ionic temperature of $2000 \mathrm{eV}$ was used to determine the Doppler width. Also, following the experimental design a 100 - $\mu$ m-radius cylindrical geometry was considered to compute the radiation transport. If we neglect the reabsorption effects and an optically thin calculation is carried out, then average charge state drops to 48.5 , just below of the experimental lower limit.

Finally, we present the analysis of an optically thick laser-produced gas xenon plasma obtained at LULI [96]. The ion density was adjusted to be $n_{i o n}=4.75 \times 10^{18} \mathrm{~cm}^{-3}$ and other measured parameters were $T_{e}=415 \pm 40 \mathrm{eV}, n_{e}$ $=(1.30 \pm 0.05) \times 10^{20} \mathrm{~cm}^{-3}$, and $\bar{Z}=27.4 \pm 1.5$. For this case two different calculations were done, in the first one we followed a relativistic DCA approach using the analyticalpotential-based atomic model (ABAKO-a) — as in the previous cases-while in the second one we used atomic data computed with FAC (ABAKO-b). In accordance with the experimental description, the effect of optical depth was introduced by assuming a $160 \mu \mathrm{m}$ path length by means of the escape factor method. An electron temperature of $450 \mathrm{eV}$ - the upper limit of the measurement error bar-was considered in the population calculation. This is done because the agreement is better than for $415 \mathrm{eV}$, as it happens with other simulations [96]. In Table VII we compare our calculations with the measured average ionization and the one obtained by AVERROES [96]. We also include the value obtained by using the SOSA method and fitting the recorded $M$-shell $4 f$ $\rightarrow 3 d$ spectral features adjusting the ion populations with a least-squares procedure [96]. As we can see, both two values obtained with ABAKO are comparable with AVERROES and agree well with the experimental measurement. Nevertheless, the ABAKO-b result is closer to the experiment than ABAKO-a due to the higher accuracy of the atomic data source. In Fig. 6 we show the corresponding curves for the fractional ion populations. Both ABAKO calculations are similar to the

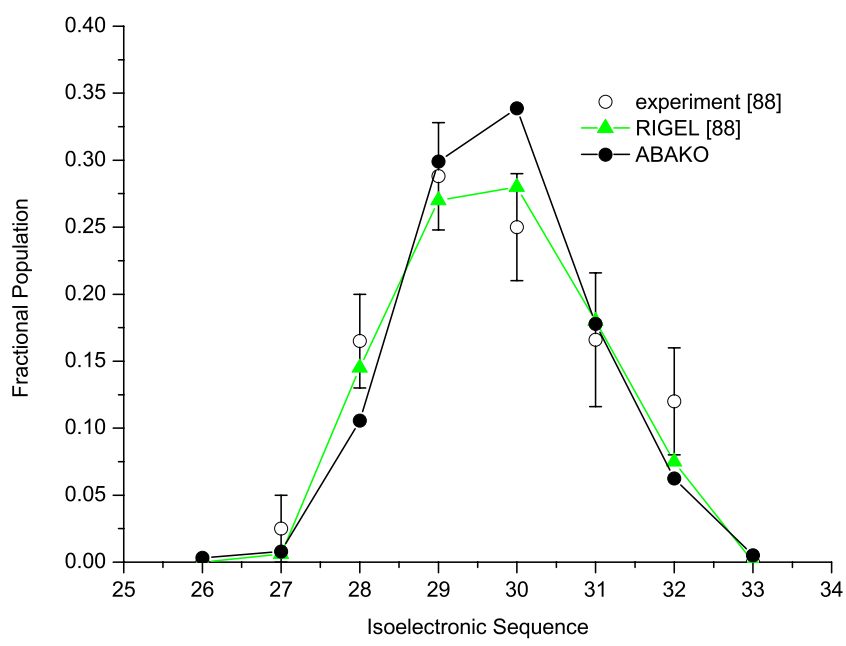

FIG. 5. (Color online) Comparison of charge state distributions simulated by different models and the measurements for the $\mathrm{Au}$ experiment with $n_{e}=6 \times 10^{20} \mathrm{~cm}^{-3}$ and $T_{e}=2200 \mathrm{eV}$ performed at NOVA.
TABLE VI. Average ionization for the Au experiment performed on NOVA at LLNL [94] and calculations obtained by different models assuming $n_{e}=6 \times 10^{20} \mathrm{~cm}^{-3}$ and $T_{e}=2200 \mathrm{eV}$.

\begin{tabular}{lc}
\hline \hline Source & $\bar{Z}$ \\
\hline Experiment [94] & $49.3 \pm 0.5$ \\
ABAKO & 49.2 \\
AVERROES [95] & 49.6 \\
FLYCHK [25] & 49.6 \\
RIGEL [94] & 49.1 \\
\hline \hline
\end{tabular}

AVERROES result, while the SOSA fit shows a different behavior. Probably this is because of the electron temperature assumed in the fit, which was $400 \mathrm{eV}$. Additionally, an analysis of the xenon X-ray emission spectrum based on ABAKO population kinetics was accomplished in [80].

As a conclusion, a good agreement with the experimental data has been found in general for all above high- $Z$ plasmas involving $M$-shell ions, which demonstrates the utility of our model to perform kinetics calculations for such large and complex cases. ABAKO has also been utilized for the spectroscopic diagnosis of the electron temperature and density by means of analysis and modeling of the plasma $K$-shell emission spectrum. In this regard, we discuss here two recent applications. The first one is the simulation of a series of experiments of laser-produced dense and hot aluminum plasmas performed at LULI $[97,98]$. The experimental setup allowed us to extract from the recorded image a succession of time-integrated spatially resolved spectral traces, each of which associated with the emission coming from a different slice of the aluminum plasma along the laser-target axis. Further details related to the experiment can be found in [98]. For this application, we used ABAKO to compute a database of emergent intensities in the photon energy range from 1800 to $2300 \mathrm{eV}$ over a $20 \times 20$ grid of $T_{e}$ and $n_{e}$ in the domain of interest, i.e., $300-500 \mathrm{eV}$ and $10^{21}-10^{23} \mathrm{~cm}^{-3}$. The escape factor model for slab geometry was used to take into account opacity effects in the kinetics. Particularly for these calculations an $80-\mu \mathrm{m}$-thick aluminum plasma was assumed, which matches with the thickness of the aluminum layer of the target analyzed here. We computed the atomic data using FAC, thus satisfying the required accuracy for spectroscopic

TABLE VII. Average ionization for the Xe plasma created at LULI [96] and calculations obtained by different models. For ABAKO and AVERROES the ion density $n_{\text {ion }}=4.75 \times 10^{18} \mathrm{~cm}^{-3}$ and $T_{e}=450 \mathrm{eV}$ are input parameters. For the SOSA fit a value of $T_{e}$ $=400 \mathrm{eV}$ was assumed.

\begin{tabular}{lc}
\hline \hline Source & $\bar{Z}$ \\
\hline Experiment [96] & $27.4 \pm 1.5$ \\
ABAKO-a & 26.6 \\
ABAKO-b & 27.1 \\
AVERROES [96] & 26.8 \\
SOSA fit [96] & 26.5 \\
\hline
\end{tabular}




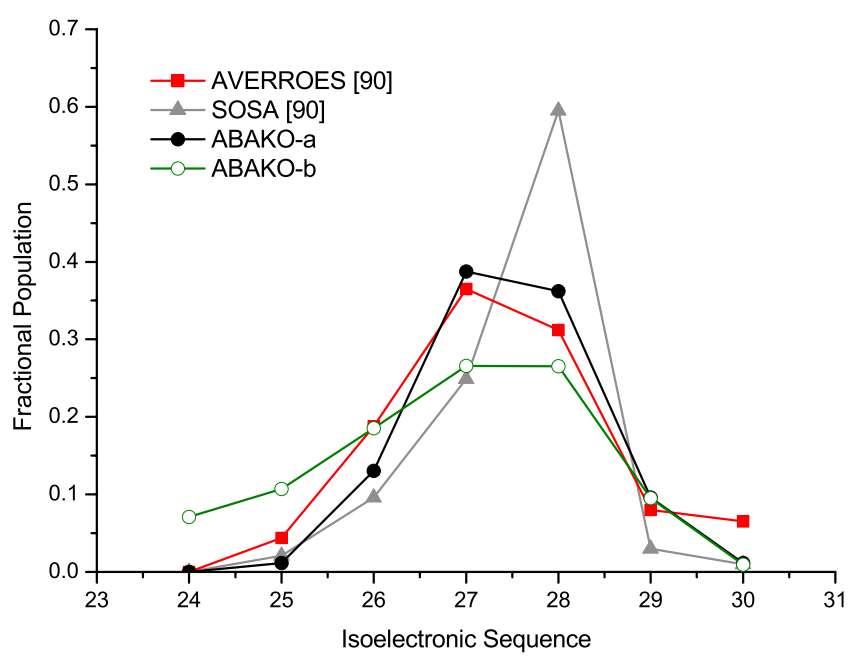

FIG. 6. (Color online) Comparison of charge state distributions simulated by different models for the Xe experiment performed at LULI.

diagnostics. We used a semiempirical formula for an estimate of Stark widths [76]. Then, natural, Stark, and Doppler broadenings were included in the context of Voigt line profiles. Complete redistribution was assumed and line overlapping was considered. The determination of $T_{e}$ and $n_{e}$ for a given spectral trace was performed by searching in the database the synthetic spectrum that yields the best fit to the data in the sense of a least-squares minimization. An example of the simulated spectra is given in Fig. 7. A systematic application of this procedure could be useful to determine timeintegrated spatially resolved profiles of $n_{e}$ and $T_{e}$.

A second application is related to the observation of argon $K$-shell line spectra from argon-doped deuterium-filled direct-drive implosion cores [99,100]. The experiments were performed at the Laboratory for Laser Energetics at the University of Rochester. Emission from the argon tracer becomes intense at the collapse of the implosion when the deuterium gas reaches keV-range electron temperatures and the electron density reaches the order of magnitude of $10^{24} \mathrm{~cm}^{-3}$. The argon emission includes the He $\alpha$, Ly $\alpha$, He $\beta$, He $\gamma$, Ly $\beta$, and Ly $\gamma$ line emissions and their associated $\mathrm{He}$ - and Li-like satellites thus covering a broad photon energy range from 3100 to $4300 \mathrm{eV}$ approximately. The time-dependent Ar $K$-shell spectrum was monitored using time-resolved $\mathrm{x}$-ray spectroscopy. For this case, an ABAKO model for argon was constructed that includes up to 4592 energy levels (one fully stripped, $100 \mathrm{H}$-like, $352 \mathrm{He}$-like, 519 Li-like, 644 Be-like, 1299 B-like, and 1677 C-like Ar). Energy levels and radiative line transition rates were computed using the atomic structure code FAC including UTA and configuration interaction corrections. The calculations take into account all nonautoionizing and autoionizing states characterized by principal quantum numbers consistent with the continuum lowering, so that the actual final number of levels included in the calculation depends on the plasma conditions. For the temperature and density conditions studied here this means that states with principal quantum number $n$ up to 4 or 5 are typically considered in the calculation, including the effects of high-order satellites that overlap and
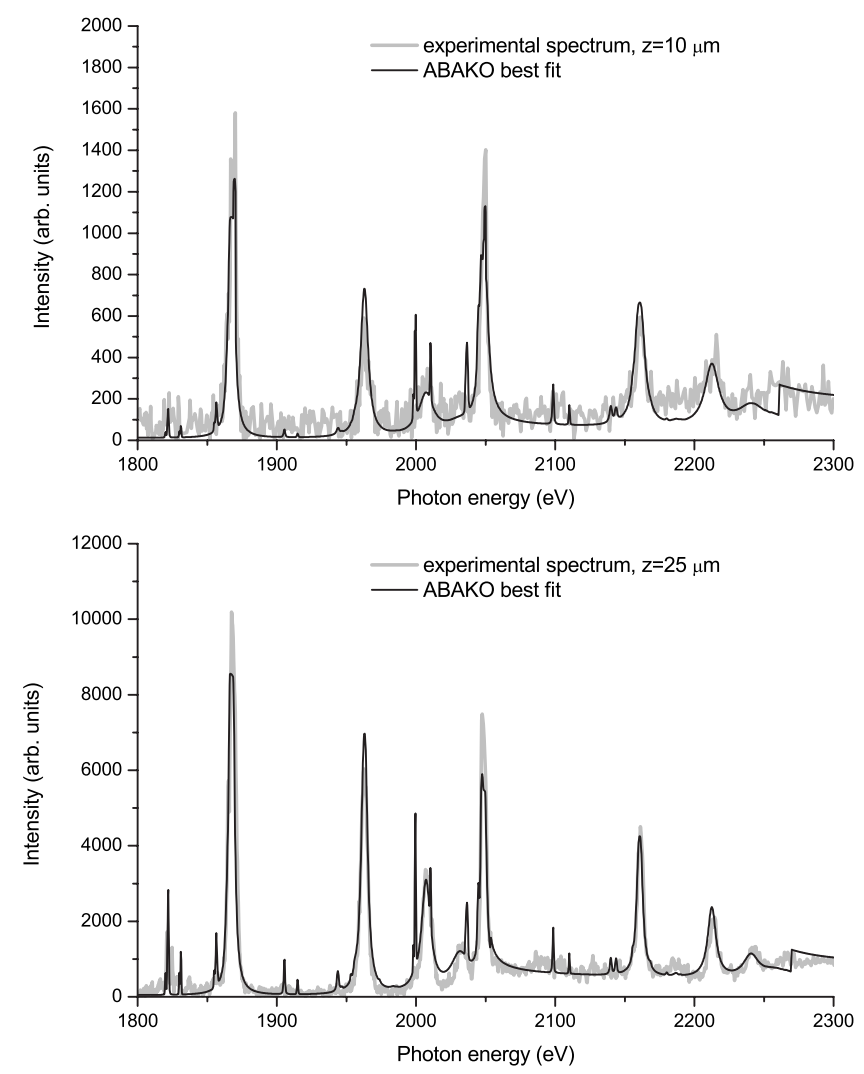

FIG. 7. Comparison between an $\mathrm{Al} K$-shell spectrum recorded at LULI and the ABAKO best fit. Two examples are shown corresponding to different recorded measurements along the laser-target axis. The analysis gives (top) $n_{e}=2.26 \times 10^{22} \mathrm{~cm}^{-3}$ and $T_{e}=469 \mathrm{eV}$ and (bottom) $n_{e}=1.52 \times 10^{22} \mathrm{~cm}^{-3}$ and $T_{e}=417 \mathrm{eV}$.

blend with the parent line transition, thus affecting both the emissivity and the opacity of the composite spectral feature [101]. Radiation transport effects in the atomic kinetics due to line trapping in the plasma are taken into account via escape factors, and the emergent line intensity distribution is computed using an analytical integration of the radiation transport equation for the case of a uniform spherical plasma source [102]. The line shapes employed in the calculation of the escape factors are Voigt line profiles in which the width of the Lorentzian contribution considers natural and an approximate Stark width [76] and the width of the Gaussian contribution characterize the thermal Doppler broadening. On the other hand, the intrinsic line shapes used to transport the line radiation through the plasma are detailed Stark broadened line profiles. To this end, a database of line profiles was computed for line transitions arising from nonautoionizing as well as autoionizing states including the broadening effects due to plasma electron and ion microfields, as well as natural and Doppler broadenings [103,104]. Furthermore, since deuterium ions are the dominant type of perturbing ion in the plasma core, ion dynamics effects were also considered in the Stark line broadening calculation.

As an illustration, Fig. 8 shows a comparison between two different time-resolved spectra extracted from the OMEGA shots 49952 and 49954 and the corresponding best fits obtained with ABAKO. In this case we calculated a data- 

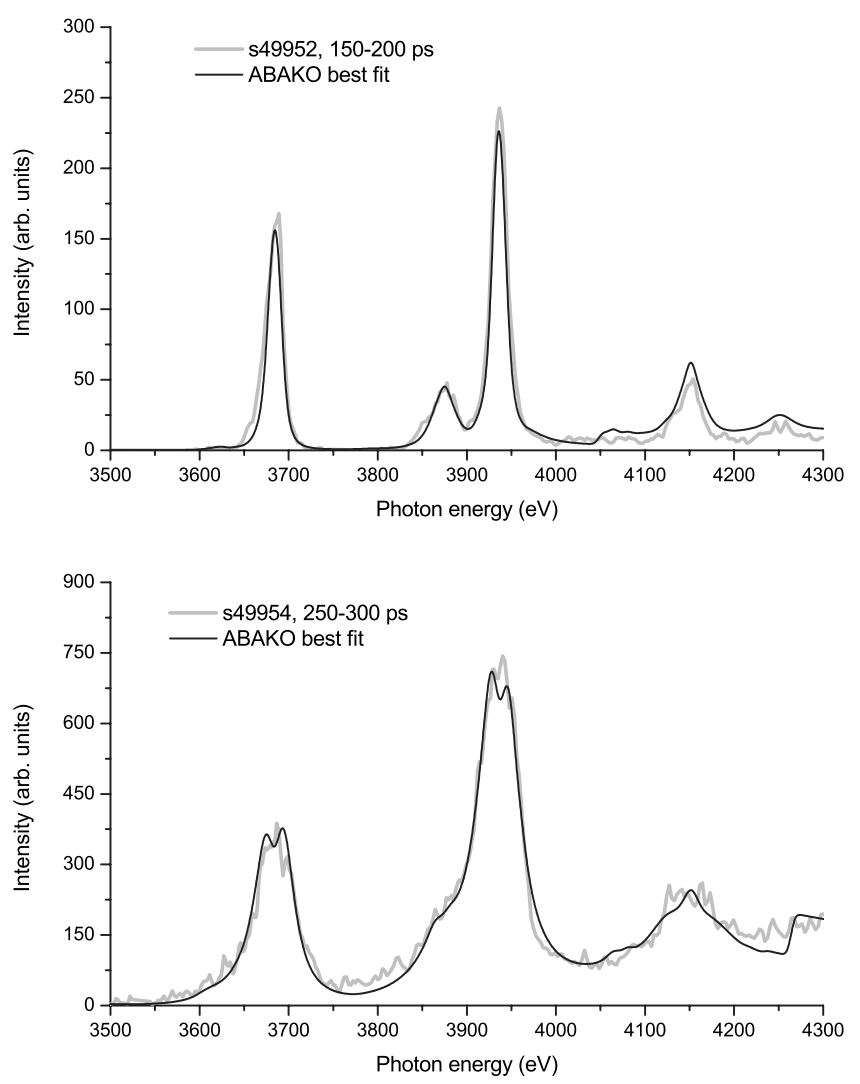

FIG. 8. Comparison between a time-resolved Ar $K$-shell spectrum and the ABAKO best fit: (top) OMEGA shot 49952, the extracted conditions are $T_{e}=1412 \mathrm{eV}$ and $n_{e}=2.81 \times 10^{23} \mathrm{~cm}^{-3}$; (bottom) OMEGA shot 49954, the extracted conditions are $T_{e}$ $=1518 \mathrm{eV}$ and $n_{e}=1.51 \times 10^{24} \mathrm{~cm}^{-3}$.

base of simulated intensities in the photon energy range from 3500 to $4300 \mathrm{eV}$ over a $35 \times 65$ grid of electron temperature (from 500 up to $2250 \mathrm{eV}$ ) and density (from $3 \times 10^{22}$ up to $5 \times 10^{24} \mathrm{~cm}^{-3}$ ) values. Before comparing with data, each synthetic spectrum was convolved with a Gaussian function of full width at half maximum $=9 \mathrm{eV}$ in order to take into account the instrumental broadening effect. As in the previous application, a search based on a weighted least-squares minimization comparison between experimental and theoretical spectra leads to a single absolute minimum from which the best fit and the density and temperature in the core are extracted. The goodness of the fit is assessed by the value of the weighted $Q^{2}$ divided by the number of degrees of freedom, which is of order 1 . Work is in progress to use this analysis for a systematic obtaining of the electron temperature and density time histories through the collapse of the implosion. Also, the argon bound-free emission observed in the high photon energy range of the recorded spectra and the effect of the continuum lowering in argon-deuterium mixtures are being investigated [105].

\section{CONCLUSIONS}

In this paper we have presented ABAKO, a versatile CR model, to determine the population distribution of atomic levels and radiative properties of steady-state NLTE plasmas. Special care was taken during the ABAKO development to achieve an optimal balance between accuracy and computational cost. ABAKO combines a set of simple analytical models which yield a substantial saving of computational requirements, but providing satisfactory results in relation to those obtained from more elaborate codes and experimental data. ABAKO has a built-in atomic model that allows us to calculate DCA atomic data using an analytical potential, although the use of external atomic data is also possible. Furthermore, analytical expressions have been employed to determine the rate coefficients of the atomic processes included in the $\mathrm{CR}$ model. In particular, a known approximation consisting of calculating the electron capture cross section in terms of the collisional excitation cross section has been adapted to work in a DCA scheme. This allows autoionizing states, whose contribution has been shown critical to determine the ionization balance, to be explicitly included in the kinetics in a fast and efficient way. The screening effects due to neighboring electrons and ions in the plasma are described in terms of a lowering of the ionization potentials, following the Stewart and Pyatt model. We would like to note at this point that ABAKO involves a full coupling between autoionizing and nonautoionizing energy levels within the same ion, but also between those belonging to neighboring ions; thereby any recombination cascade consistent with the radiative selection rules and continuum lowering is automatically taken into account. Self-absorption effects in uniform plasmas are included via escape factors for the three basic geometries - planar, cylindrical, and spherical.

Since the determination of level population distributions often involves very large sparse CR matrices, iterative methods are used to perform the matrix inversion because they typically require less memory and can yield an approximation to the solution significantly faster than a direct method. Considering all the characteristics described above, ABAKO results a versatile $\mathrm{CR}$ model that can be applied to low-tohigh $Z$ ions with a variety of laboratory plasma conditions: coronal, LTE or NLTE, and optically thin or thick plasmas.

Regarding the computer hardware requirements, $\mathrm{ABAKO}$ runs on standard workstations. Specifically, the results discussed in this paper were run in a workstation with a 2.4 $\mathrm{GHz}$ single-core microprocessor and $2 \mathrm{~Gb}$ of random access memory. Typical execution times depend on the size of the problem, taking a few minutes for a total number of energy levels of a few thousands up to several hours when the system is on the order of 50000 or more. At present time, we are working on a parallelized version of the code and the design of a web user interface to provide online execution. As soon as the web-based interface is set up, a proper user manual will become available.

A large number of experimental and modeling comparisons show that ABAKO provides reasonable estimates of the ionization balance for a wide range of plasma temperatures and densities. In this work, besides presenting results based on computational tests proposed in the NLTE workshops, ABAKO has been used to simulate the charge state distribution of a low-density gold plasma created at EBIT-II at LLNL, a xenon gas jet plasma obtained at LULI, and a gold plasma produced at the NOVA laser. Population kinetics of 
these plasmas is hard to model because the ionization balance is globally dominated by the $M$-shell ions between $\mathrm{Cr}$ like and $\mathrm{Kr}$-like. Still, in all cases analyzed the average ionization predicted by ABAKO shows a well agreement with data and the ion population distribution compares with the experiments within no more than one charge state. ABAKO results are competitive with those obtained from more elaborate codes, also improving the results provided by codes with similar characteristics. Lastly, we used ABAKO to simulate the $K$-shell emission of a laser-produced aluminum plasma obtained at LULI. Also, a spectroscopic analysis of the argon $K$-shell emission spectra is being employed to investigate the evolution in time of OMEGA direct-drive implosion cores.

Most of the experiments above were chosen because they constitute a set of well-established benchmark cases to test the ABAKO capabilities; however, there are no restrictions for using ABAKO to perform other studies, e.g., the atomic kinetics modeling of different plasma x-ray lasers or EUV sources. An example of such an application will be published elsewhere.

Although ABAKO actually constitutes a helpful tool for many applications and research areas, its development still continues. Some improvements already underway include the extension to non-Maxwellian electron distributions and the explicit consideration of atomic processes driven by an external radiation field. Modeling of time-dependent population kinetics is another short-term goal. Also, the proposed formalism to calculate the autoionization and electron capture rate coefficient needs to be revised for the case of high- $Z$ plasmas at very high temperatures, because we have found that it leads to anomalous high values of the average ionization. Finally, another goal is the implementation of a radiation transport formalism based on zone-to-zone radiative coupling coefficients that can be applied on nonuniform plasma and can allow us to perform large-scale kinetics calculations.

\section{ACKNOWLEDGMENTS}

The authors gratefully acknowledge Dr. James Colgan (Theoretical Division, Los Alamos National Laboratory) for providing the ATOMIC calculation data for comparisons and for helpful discussion on carbon cases. This work was supported by Research Grant No. ENE2008-06668-C02/FTN of the Spanish Ministry of Science and Innovation and also by the "Keep in Touch" Project of the EU.
[1] H. K. Chung and R. W. Lee, High Energy Density Phys. 5, 1 (2009).

[2] D. R. Bates, A. E. Kingston, and R. W. P. McWhirter, Proc. R. Soc. London, Ser. A 267, 297 (1962).

[3] R. W. P. McWhirter, Phys. Rep. 37, 165 (1978).

[4] D. R. Bates, A. E. Kingston, and R. W. P. McWhirter, Proc. R. Soc. London, Ser. A 270, 155 (1962).

[5] S. Mazevet and J. Abdallah, J. Phys. B 39, 3419 (2006).

[6] C. J. Fontes, J. Colgan, H. L. Zhang, and J. Abdallah, Jr., J. Quant. Spectrosc. Radiat. Transf. 99, 175 (2006).

[7] A. Bar-Shalom, M. Klapisch, and J. Oreg, J. Quant. Spectrosc. Radiat. Transf. 71, 169 (2001).

[8] Y. V. Ralchenko and Y. Maron, J. Quant. Spectrosc. Radiat. Transf. 71, 609 (2001).

[9] S. B. Hansen, Ph.D. thesis, University of Nevada, Reno, 2003.

[10] O. Peyrusse, Nucl. Fusion 44, S202 (2004).

[11] O. Peyrusse, J. Phys. B 32, 683 (1999).

[12] M. Poirier, J. Phys. B 41, 025701 (2008).

[13] A. Bar-Shalom, J. Oreg, and M. Klapisch, Phys. Rev. E 56, R70 (1997).

[14] J. Bauche, C. Bauche-Arnoult, and M. Klapisch, Adv. At. Mol. Phys. 23, 131 (1987).

[15] J. Bauche, C. Bauche-Arnoult, and M. Klapisch, J. Phys. B 24, 1 (1991)

[16] A. Bar-Shalom, J. Oreg, W. H. Goldstein, D. Shvarts, and A. Zigler, Phys. Rev. A 40, 3183 (1989).

[17] O. Peyrusse, J. Phys. B 33, 4303 (2000).

[18] A. Bar-Shalom, J. Oreg, and M. Klapisch, J. Quant. Spectrosc. Radiat. Transf. 65, 43 (2000).

[19] J. Bauche, C. Bauche-Arnoult, and K. B. Fournier, Phys. Rev. E 69, 026403 (2004).
[20] S. B. Hansen, J. Bauche, C. Bauche-Arnoult, and M. F. Gu, High Energy Density Phys. 3, 109 (2007).

[21] J. Abdallah, Jr. and M. E. Sherrill, High Energy Density Phys. 4, 124 (2008).

[22] M. Busquet, Phys. Fluids B 5, 4191 (1993).

[23] A. F. Nikiforov, V. G. Novikov, V. B. Uvarov, V. V. Dragalov, and A. D. Solomyannaya, Third International Opacity Workshop Code Comparison Study, WorkOp-III (Max-Planck Institute fur Quantenoptik, Garching, Germany, 1995).

[24] G. Faussurier, C. Blancard, and E. Berthier, Phys. Rev. E 63, 026401 (2001)

[25] H. K. Chung, M. H. Chen, W. L. Morgan, Y. Ralchenko, and R. W. Lee, High Energy Density Phys. 1, 3 (2005).

[26] D. Mihalas, Stellar Atmospheres (W. H. Freeman and Company, San Francisco, CA, 1970).

[27] J. P. Apruzese, J. Davis, K. G. Whitney, J. W. Thornhill, P. C. Kepple, and R. W. Clark, Phys. Plasmas 9, 2411 (2002).

[28] D. Mostacci, L. M. Montierth, J. P. Dinguirard, and R. L. Morse, Phys. Fluids B 1, 2106 (1989).

[29] D. C. Eder and H. A. Scott, J. Quant. Spectrosc. Radiat. Transf. 45, 189 (1991).

[30] O. Peyrusse, Phys. Fluids B 4, 2007 (1992).

[31] I. Hubeny and T. Lanz, Astrophys. J. 439, 875 (1995).

[32] Methods in Radiative Transfer, edited by W. Kalkofen (Cambridge University Press, Cambridge, England, 1984).

[33] G. L. Olson and P. B. Kunasz, J. Quant. Spectrosc. Radiat. Transf. 38, 325 (1987).

[34] H. A. Scott, J. Quant. Spectrosc. Radiat. Transf. 71, 689 (2001).

[35] F. E. Irons, J. Quant. Spectrosc. Radiat. Transf. 22, 1 (1979).

[36] F. E. Irons, J. Quant. Spectrosc. Radiat. Transf. 22, 21 (1979).

[37] F. E. Irons, J. Quant. Spectrosc. Radiat. Transf. 22, 37 (1979). 
[38] J. P. Apruzese, J. Davis, D. Duston, and K. G. Whitney, J. Quant. Spectrosc. Radiat. Transf. 23, 479 (1980).

[39] J. P. Apruzese, J. Quant. Spectrosc. Radiat. Transf. 25, 419 (1981).

[40] J. P. Apruzese, J. Quant. Spectrosc. Radiat. Transf. 34, 447 (1985).

[41] C. Bowen, A. Decoster, C. J. Fontes, K. B. Fournier, O. Peyrusse, and Y. V. Ralchenko, J. Quant. Spectrosc. Radiat. Transf. 81, 71 (2003).

[42] C. Bowen, R. W. Lee, and Y. Ralchenko, J. Quant. Spectrosc. Radiat. Transf. 99, 102 (2006).

[43] J. G. Rubiano, R. Florido, C. Bowen, R. W. Lee, and Y. Ralchenko, High Energy Density Phys. 3, 225 (2007).

[44] C. J. Fontes, J. Abdallah, Jr., C. Bowen, R. W. Lee, and Y. Ralchenko, High Energy Density Phys. 5, 15 (2009).

[45] P. Martel, L. Doreste, E. Mínguez, and J. M. Gil, J. Quant. Spectrosc. Radiat. Transf. 54, 621 (1995).

[46] J. M. Gil, P. Martel, E. Mínguez, J. G. Rubiano, R. Rodríguez, and F. H. Ruano, J. Quant. Spectrosc. Radiat. Transf. 75, 539 (2002).

[47] R. Rodriguez, J. M. Gil, and R. Florido, Phys. Scr. 76, 418 (2007).

[48] R. Rodríguez, J. G. Rubiano, J. M. Gil, P. Martel, E. Mínguez, and R. Florido, J. Quant. Spectrosc. Radiat. Transf. 75, 723 (2002).

[49] J. G. Rubiano, R. Florido, R. Rodríguez, J. M. Gil, P. Martel, and E. Mínguez, J. Quant. Spectrosc. Radiat. Transf. 83, 159 (2004).

[50] A. K. Rajagopal, Adv. Chem. Phys. 41, 59 (1980).

[51] Y. Ralchenko, J. Quant. Spectrosc. Radiat. Transf. 99, 499 (2006).

[52] R. D. Cowan, The Theory of Atomic Structure and Spectra (University of California Press, Berkeley, CA, 1981); ftp:// aphysics.lanl.gov/pub/cowan/

[53] M. F. Gu, 14th APS Topical Conference on Atomic Processes in Plasmas (AIP, New York, 2004), pp. 127-136.

[54] S. J. Rose, J. Phys. B 25, 1667 (1992).

[55] J. C. Stewart and K. D. Pyatt, Astrophys. J. 144, 1203 (1966).

[56] R. M. More, LLNL Technical Report No. UCRL-84991, 1981 (unpublished).

[57] H. Van Regemorter, Astrophys. J. 136, 906 (1962).

[58] R. Mewe, Astron. Astrophys. 20, 215 (1972).

[59] D. H. Sampson and H. L. Zhang, Phys. Rev. A 45, 1556 (1992).

[60] L. B. Golden and D. H. Sampson, Astrophys. J. 170, 181 (1971).

[61] W. Lotz, Z. Phys. 206, 205 (1967).

[62] A. Burgess and M. C. Chidichimo, Mon. Not. R. Astron. Soc. 203, 1269 (1983).

[63] D. L. Moores, L. B. Golden, and D. H. Sampson, J. Phys. B 13, 385 (1980).

[64] L. B. Golden and D. H. Sampson, J. Phys. B 13, 2645 (1980).

[65] V. A. Bernshtam, Y. V. Ralchenko, and Y. Maron, J. Phys. B 33, 5025 (2000).

[66] H. A. Kramers, Philos. Mag. 46, 836 (1923).

[67] Z. Q. Wu, B. A. Zhang, and Y. B. Qiu, Chin. Phys. 9, 519 (2000).

[68] J. R. Albritton and B. G. Wilson, Phys. Rev. Lett. 83, 1594 (1999).

[69] V. L. Jacobs, J. Quant. Spectrosc. Radiat. Transf. 54, 195
(1995).

[70] R. Doron, E. Behar, P. Mandelbaum, and J. L. Schwob, J. Quant. Spectrosc. Radiat. Transf. 65, 161 (2000).

[71] J. Bauche, C. Bauche-Arnoult, and O. Peyrusse, High Energy Density Phys. 5, 51 (2009).

[72] V. L. Jacobs, High Energy Density Phys. 5, 80 (2009).

[73] A. Burgess, Astrophys. J. 139, 776 (1964).

[74] H. R. Griem, Principles of Plasma Spectroscopy (Cambridge University Press, Cambridge, England, 1997).

[75] R. C. Mancini, R. F. Joyce, and C. F. Hooper, Jr., J. Phys B 20, 2975 (1987).

[76] M. S. Dimitrijevic and N. Konjevic, Astron. Astrophys. 172, 345 (1987).

[77] R. Florido, Ph.D. thesis, Universidad de Las Palmas de Gran Canaria, Las Palmas de Gran Canaria, 2007.

[78] R. C. Mancini (unpublished).

[79] Y. Saad, Iterative Methods for Sparse Linear Systems (SIAM, Philadelphia, PA, 2003).

[80] R. Rodríguez, R. Florido, J. M. Gil, J. G. Rubiano, P. Martel, and E. Mínguez, Laser Part. Beams 26, 433 (2008).

[81] E. Mínguez, R. Florido, R. Rodríguez, J. M. Gil, J. G. Rubiano, M. A. Mendoza, D. Suárez, and P. Martel, High Energy Density Phys. (in press).

[82] R. Rodríguez, R. Florido, J. M. Gil, J. G. Rubiano, P. Martel, and E. Mínguez, J. Quant. Spectrosc. Radiat. Transf. 110, 2191 (2009).

[83] R. W. Lee, J. K. Nash, and Y. Ralchenko, J. Quant. Spectrosc. Radiat. Transf. 58, 737 (1997).

[84] R. Florido, J. M. Gil, R. Rodriguez, J. G. Rubiano, P. Martel, and E. Mínguez, 28th European Conference on Laser Interaction with Matter (C. R. ENEA, Frascati, Italy, 2004), pp. 389 413.

[85] H. K. Chung, M. H. Chen, and R. W. Lee, High Energy Density Phys. 3, 57 (2007).

[86] D. Salzmann, Atomic Physics in Hot Plasmas (Oxford University Press, Oxford, 1998).

[87] J. M. Gil, R. Rodríguez, R. Florido, J. G. Rubiano, P. Martel, and E. Mínguez, Laser Part. Beams 26, 21 (2008).

[88] J. Colgan, C. J. Fontes, and J. Abdallah, Jr., High Energy Density Phys. 2, 90 (2006).

[89] M. Poirier and F. de Gaufridy de Dortan, J. Appl. Phys. 101, 063308 (2007).

[90] A. Burgess, Astrophys. J. 141, 1588 (1965).

[91] A. L. Merts, R. D. Cowan, and N. H. Magee, Jr., Los Alamos National Laboratory Technical Report No. LA-6220-MS, 1976 (unpublished).

[92] K. L. Wong, M. J. May, P. Beiersdorfer, K. B. Fournier, B. Wilson, G. V. Brown, and P. Springer, Phys. Rev. Lett. 90, 235001 (2003).

[93] O. Peyrusse, C. Bauche-Arnoult, and J. Bauche, J. Phys. B 38, L137 (2005).

[94] M. E. Foord, S. H. Glenzer, R. S. Thoe, K. L. Wong, K. B. Fournier, B. G. Wilson, and P. T. Springer, Phys. Rev. Lett. 85, 992 (2000).

[95] O. Peyrusse, J. Quant. Spectrosc. Radiat. Transf. 71, 571 (2001).

[96] C. Chenais-Popovics, V. Malka, J. C. Gauthier, S. Gary, O. Peyrusse, M. Rabec-Le Gloahec, I. Matsushima, C. BaucheArnoult, A. Bachelier, and J. Bauche, Phys. Rev. E 65, 046418 (2002). 
[97] R. Schott et al., J. Quant. Spectrosc. Radiat. Transf. 81, 441 (2003).

[98] R. Schott et al., 16th International Conference on Spectral Line Shapes (American Institute of Physics, New York, 2002), Vol. 12, pp. 340-351.

[99] R. Florido, T. Nagayama, R. C. Mancini, R. Tommasini, J. A. Delettrez, S. P. Regan, V. A. Smalyuk, R. Rodríguez, and J. M. Gil, Rev. Sci. Instrum. 79, $10 \mathrm{E} 310$ (2008).

[100] T. Nagayama, R. C. Mancini, R. Florido, R. Tommasini, J. A. Koch, J. A. Delettrez, S. P. Regan, V. A. Smalyuk, L. A. Welser-Sherrill, and I. E. Golovkin, Rev. Sci. Instrum. 79, 10E921 (2008).
[101] I. E. Golovkin and R. C. Mancini, J. Quant. Spectrosc. Radiat. Transf. 65, 273 (2000).

[102] T. J. Burris-Mog et al., J. Quant. Spectrosc. Radiat. Transf. 99, 120 (2006).

[103] L. A. Woltz and C. F. Hooper, Jr., Phys. Rev. A 38, 4766 (1988).

[104] R. C. Mancini, D. P. Kilcrease, L. A. Woltz, and C. F. Hooper, Jr., Comput. Phys. Commun. 63, 314 (1991).

[105] R. Florido, R. C. Mancini, T. Nagayama, R. Tommasini, J. A. Delettrez, S. P. Regan, V. A. Smalyuk, R. Rodríguez, and J. M. Gil, High Energy Density Phys. (in press). 